\title{
Chua's Oscillator: A Compendium of Chaotic Phenomena
}

\author{
by LADISLAV PIVKA, CHAI WAH WU and ANSHAN HUANG
}

Electronics Research Laboratory and Department of Electrical Engineering and Computer Sciences, University of California, Berkeley, CA 94720, U.S.A.

ABSTRACT: Chua's oscillator is the only real physical object known to date in which chaotic behavior has been observed experimentally and numerically, and proved rigorously. In summarizing the chaotic phenomena observed so far from the oscillator we emphasize its universality by showing how the dynamical phenomena from other $3 D$ oscillators can be reproduced by using Chua's oscillator. The possibility of its use as an elementary cell in cellular neural networks is briefly discussed.

\section{Introduction}

In the last three decades, chaotic phenomena have become a subject of great interest in different areas of physics, biology, economics, and other sciences. Once considered a rare phenomenon in nature, whose occurrence is subject to very special conditions, the accepted belief today is that chaotic phenomena permeate almost all relevant physical and biological processes.

The three standard methods of investigation were applied to the early models with chaotic dynamics numerical $(\mathbf{1})$, experimental $(2,3)$, and analytical (4-7). In view of the amount of effort invested in the research of chaos it is somewhat surprising that Chua's oscillator still remains the only real physical object in which chaos has been observed numerically and experimentally, and its robustness has been proved mathematically. This was made possible by the remarkably simple structure of the underlying circuit. Chaos is a nonlinear phenomenon, but we would like to analyze it using linear techniques which are more developed. A relatively simple circuitry makes it possible to furnish a piecewise-linear form for the nonlinearity needed to generate chaos. In fact, as little as two linear segments of the nonlinear resistor are sufficient to do the job. A transition to chaos can be initiated in several fundamentally different ways. Also, the most common form of chaotic behavior - so called chaotic attractors - can assume a multitude of shapes and can be classified according to different criteria, e.g. various types of fractal dimensions, eigenvalues of the associated equilibria, etc. Such characteristics of chaos were being introduced and studied over the past decades by using many oscillators, capable of generating certain types of chaos. It was not clear whether all known types of chaos can be generated from a single third-order oscillator until 
L. Pivka et al.

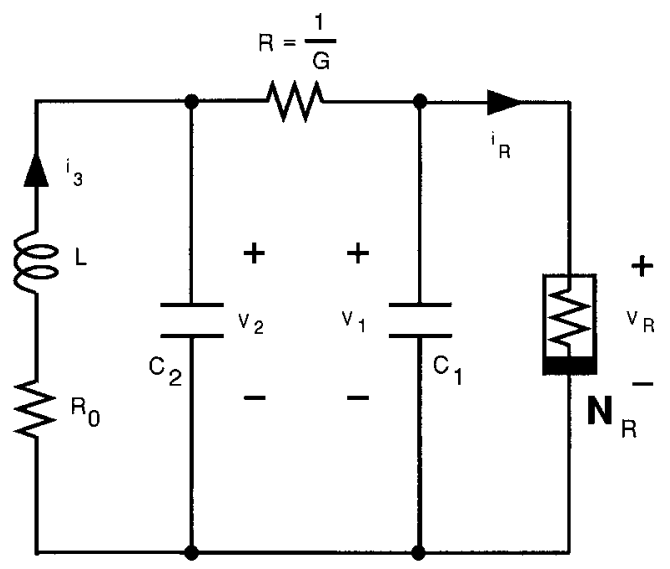

FIG. 1. Circuit diagram of Chua's oscillator.

a powerful unifying theorem (8) for 3-dimensional, piccewisc-lincar vector ficlds was proved, implying that Chua's oscillator (Fig. 1) is the most general, but structurally the simplest system capable of reproducing all possible dynamical phenomena from a certain class of $3 \mathrm{D}$ vector fields. As a consequence, Chua's oscillator can be used to mimic the behavior of other piecewise-linear oscillators and also approximate the behavior of many others which exhibit smooth nonlinearities. The corresponding theorem will be stated and proved in Section III, and examples will be given in Section IV on how Chua's oscillator can be used to reproduce the behavior from other dynamical systems. In Section II, we survey the rich variety of dynamical behaviors observed so far from Chua's oscillator. Finally, in Section $\mathrm{V}$, we take a brief view on a new, fascinating structure, the cellular neural network of Chua's oscillators, promising to spawn new and even richer phenomena with far-reaching applications.

Figure 1 shows a diagram of the oscillator, whose state equations are given by

$$
\begin{aligned}
\frac{\mathrm{d} v_{1}}{\mathrm{~d} t} & =\frac{1}{C_{1}}\left[G\left(v_{2}-v_{1}\right)-f\left(v_{1}\right)\right] \\
\frac{\mathrm{d} v_{2}}{\mathrm{~d} t} & =\frac{1}{C_{2}}\left[G\left(v_{1}-v_{2}\right)+i_{3}\right] \\
\frac{\mathrm{d} i_{3}}{\mathrm{~d} t} & =-\frac{1}{L}\left(v_{2}+R_{0} i_{3}\right),
\end{aligned}
$$

where $G=1 / R$, and $f\left(v_{1}\right)=G_{b} v_{1}+\frac{1}{2}\left(G_{a}-G_{b}\right)\left\{\left|v_{1}+E\right|-\left|v_{1}-E\right|\right\}$ is the $v-i$ characteristic of the nonlinear resistor $N_{R}$ with a slope equal to $G_{a}$ in the inner region and $G_{b}$ in the two outer regions.

By a change of variables, the state equations of Chua's oscillator (1) can be transformed into the following dimensionless form : 


$$
\left.\begin{array}{rl}
\frac{\mathrm{d} x}{\mathrm{~d} \tau} & =k \alpha(y-x-f(x)) \\
\frac{\mathrm{d} y}{\mathrm{~d} \tau} & =k(x-y+z) \\
\frac{\mathrm{d} z}{\mathrm{~d} \tau} & =k(-\beta y-\gamma z) \\
f(x) & =b x+\frac{1}{2}(a-b)\{|x+1|-|x-1|\}
\end{array}\right\}
$$

where

$$
\begin{aligned}
& x=\frac{v_{1}}{E}, \quad y=\frac{v_{2}}{E}, \quad z=i_{3}\left(\frac{R}{E}\right) \\
& \alpha=\frac{C_{2}}{C_{1}}, \quad \beta=\frac{R^{2} C_{2}}{L}, \quad \gamma=\frac{R R_{0} C_{2}}{L} \\
& a=R G_{a}, \quad b=R G_{b}, \quad \tau=\frac{t}{\left|R C_{2}\right|}, \quad \text { and } \quad \begin{array}{lll}
k=1 & \text { if } & R C_{2}>0 \\
k=-1 & \text { if } & R C_{2}<0 .
\end{array}
\end{aligned}
$$

Throughout the rest of this paper, we will mainly use the dimensionless form.

\section{Dynamical Phenomena from Chua's Oscillator}

In this section we illustrate with examples the rich variety of phenomena, especially chaotic attractors, which can be generated with Chua's oscillator. Some new phenomena, recently reported from experiments with the oscillator, are summarized in several subsections.

\subsection{Gallery of attractors}

To show the immense variety of shapes attractors can take on we reproduce those from earlier works (9) (Figs 2.1-2.22, 2.31-2.35) and add several more attractors that have been observed recently (Figs 2.23-2.30).

Table I provides the parameter values for all attractors in the figures, along with the corresponding Lyapunov exponents.

\subsection{Period-adding bifurcations}

In this phenomenon [see, for example $(\mathbf{1 0}, \mathbf{1 1})$ ], windows of consecutive periods are separated by regions of chaos. In other words, as the parameter is varied, we obtain a stable period- $n$ orbit, $n=1,2, \ldots$, followed by a region of chaos, then a stable period- $(n+1)$ orbit, followed by chaos, and then a period- $(n+2)$ orbit and so on. Examples from one such sequence are given in Fig. 3.

\subsection{Homoclinic and heteroclinic orbits in Chua's oscillator}

Closely related to the appearance of chaotic behavior in dynamical systems in general are the so-called homoclinic and heteroclinic trajectories. A homoclinic trajectory is one whose limit point in both forward and backward times is the same 
L. Pivka et al.

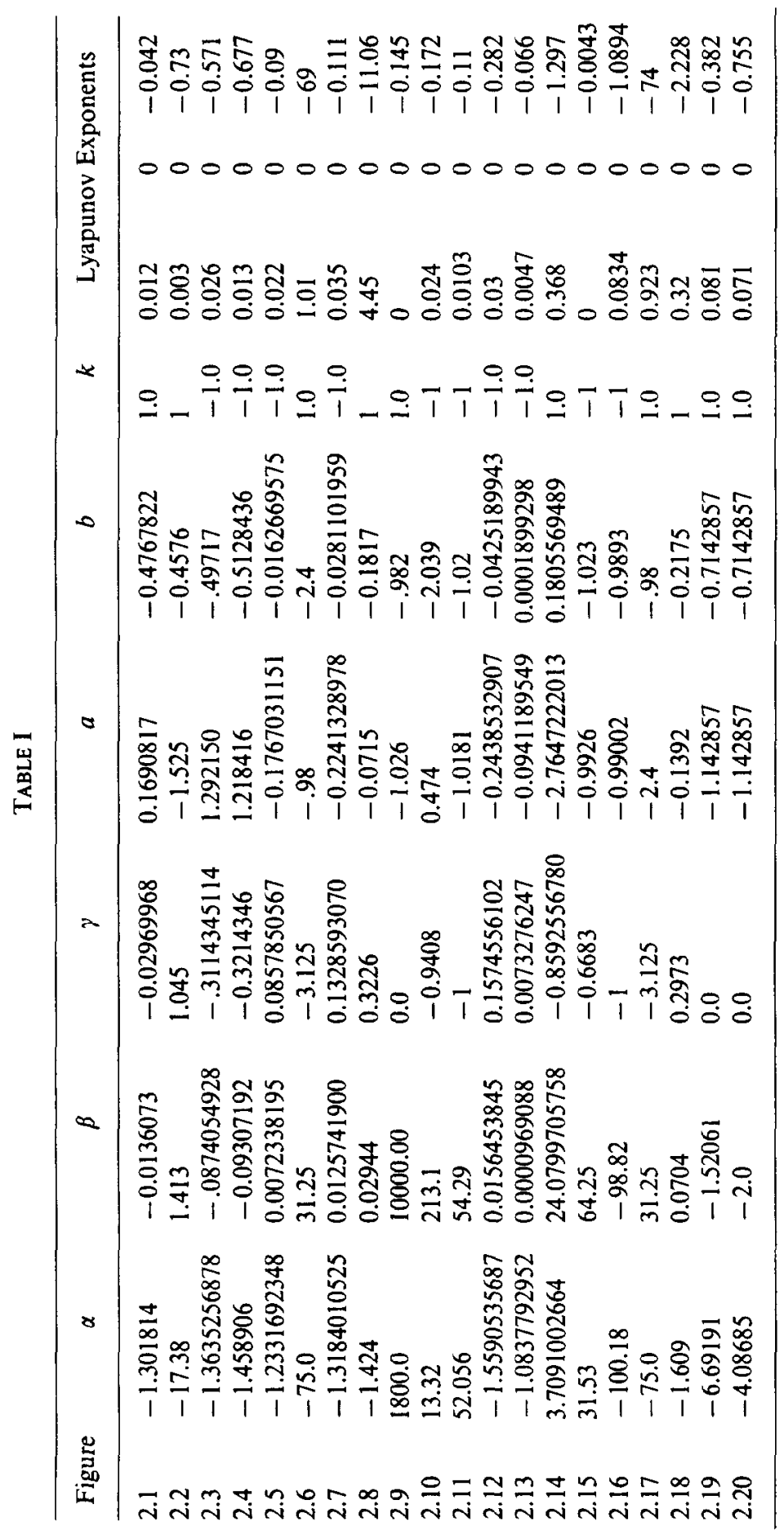




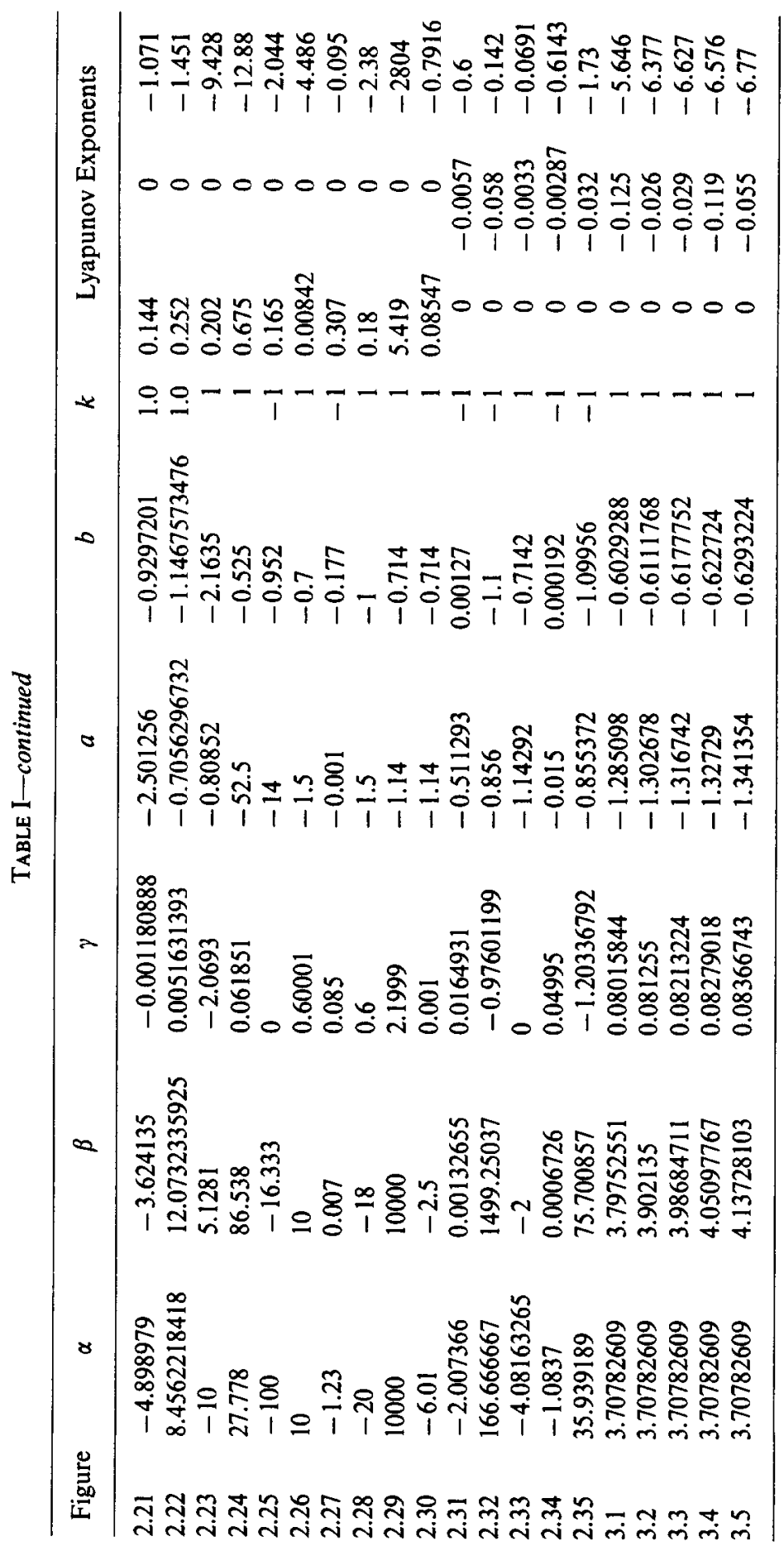




\section{Pivka et al.}
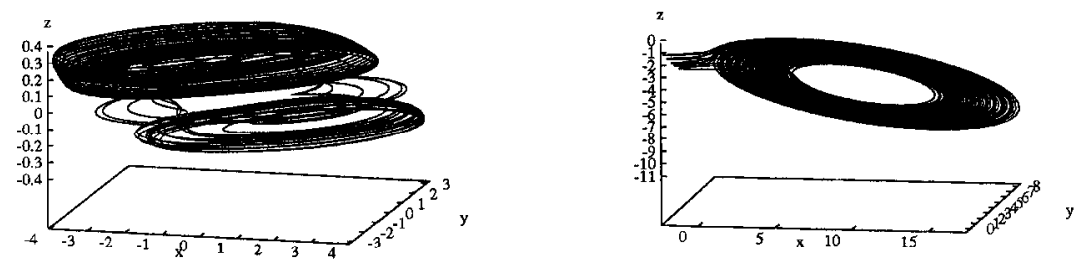

Fig. 2.1

Fig. 2.2

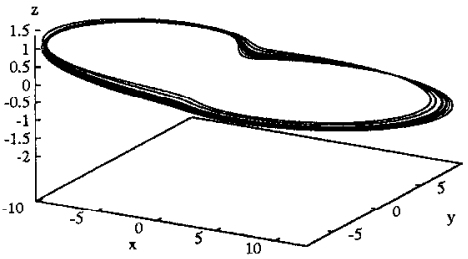

Fig. 2.3

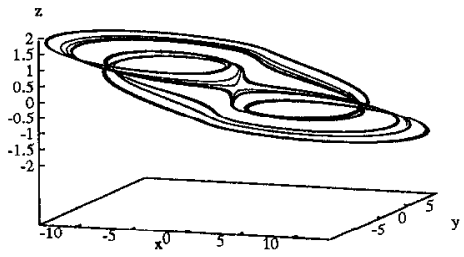

Fig. 2.4

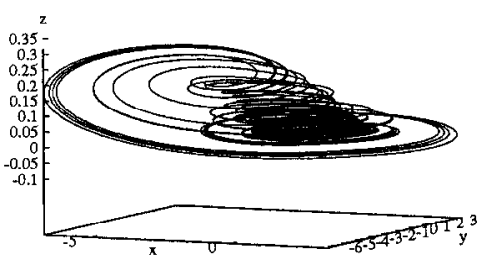

Fig. 2.5

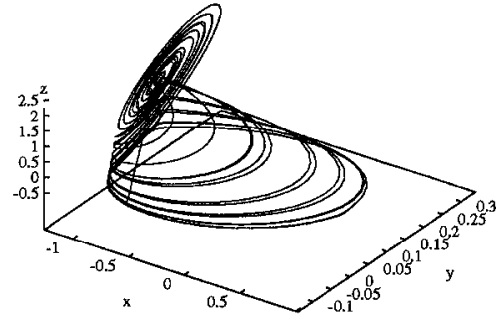

Fig. 2.6

FIG. 2. A gallery of attractors from Chua's oscillator.

saddle-type equilibrium point. On the other hand, two different equilibrium points are the limit points in forward and backward time, respectively, for a heteroclinic trajectory (see Fig. 4).

\subsection{Coexistence phenomena}

In general, for the same set of parameters, there exist many stable and unstable limit sets. The system trajectory will converge to a particular attractor if the initial conditions are chosen in the basin of attraction of the attractor. Thus which of the coexisting attractors we observe in experiments depends on the initial state of the system. Coexistence of attractors is an interesting phenomenon where the interaction of attractors can give rise to different dynamical phenomena (some of which is described in Subsection 2.6). Recently, coexistence of three distinct chaotic 

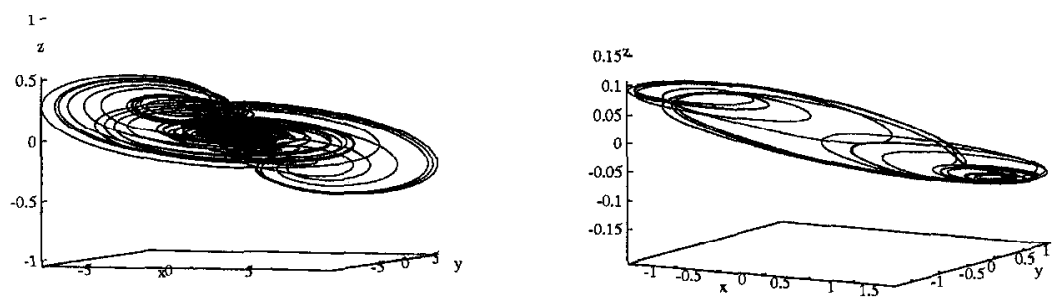

Fig. 2.7

Fig. 2.8

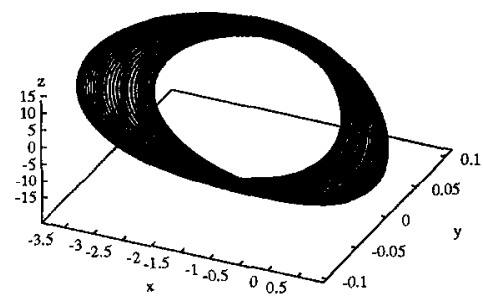

Fig. 2.9

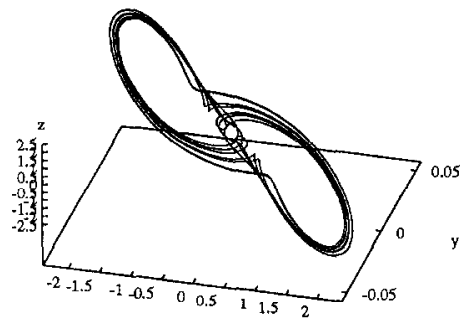

Fig. 2.11

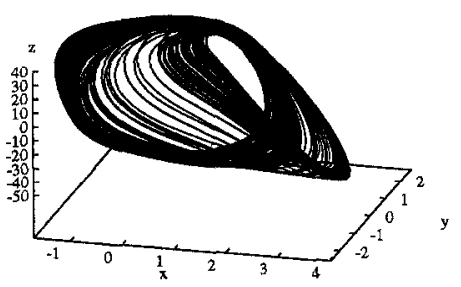

Fig. 2.10

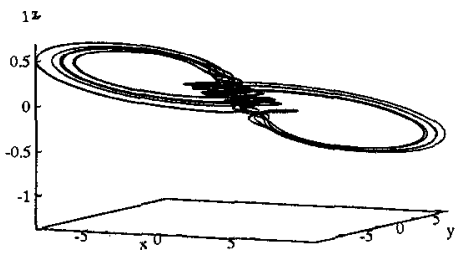

Fig. 2.12

FIG. 2 continued.

attractors has been reported in (12), where two asymmetric attractors coexist with a symmetric one (Fig. 5). Some other coexistence phenomena, including point attractors, periodic attractors, and chaotic attractors are shown in Fig. 6.

A common phenomenon in chaotic systems is the coexistence of nonstable orbits near chaotic attractors. Figure 7 illustrates such coexistence with the double scroll attractor.

\subsection{Routes to chaos}

2.5.1. Period-doubling route to chaos. When the parameter $\alpha$ is changed, an equilibrium point loses its stability and a stable limit cycle emerges through an 


\section{Pivka et al.}
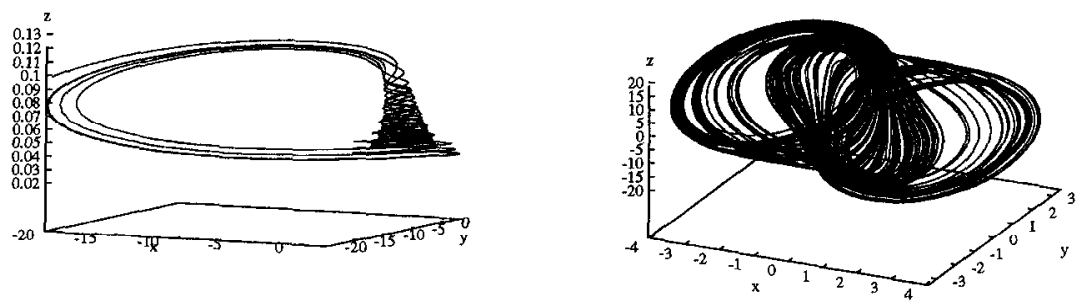

Fig. 2.13

Fig. 2.14
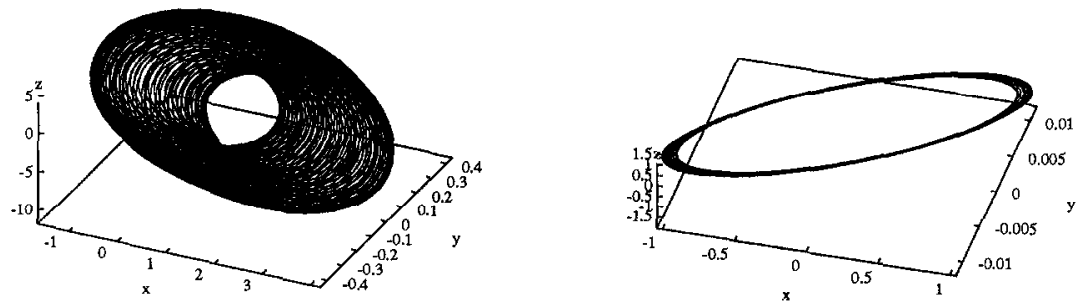

Fig. 2.15

Fig. 2.16
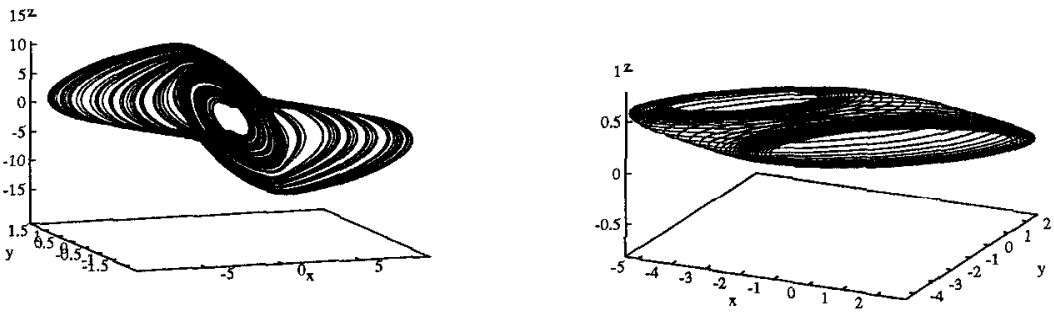

Fig. 2.17

Fig. 2.18

Fig. 2 - continued.

Andronov-Hopf bifurcation. As the parameter is changed further, the stable limit cycle eventually loses stability, and a stable limit cycle of approximately twice the period emerges, which is usually referred to as a period-2 limit cycle. Similarly a period-4 limit cycle appears after the period-2 limit cycle loses its stability. This bifurcation occurs infinitely many times at ever-decreasing intervals of the parameter range, which converges at a geometric ratc, detcrmined by the well-known Feigenbaum constant, to a limit (bifurcation point) at which point chaos is observed. This is called a period-doubling route to chaos, an example of which is shown in Fig. 8.

2.5.2. Torus breakdown route to chaos. In this route to chaos the system undergoes several Andronov-Hopf bifurcations. After two Andronov-Hopf bifurcations, we 


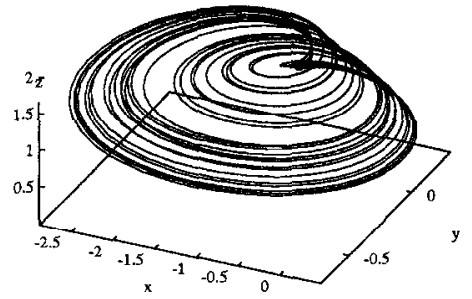

Fig. 2.19

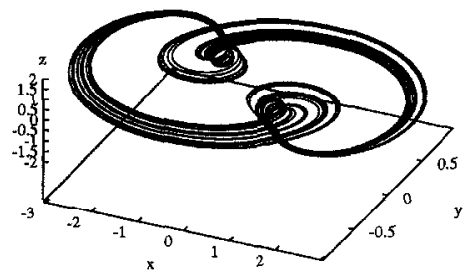

Fig. 2.20

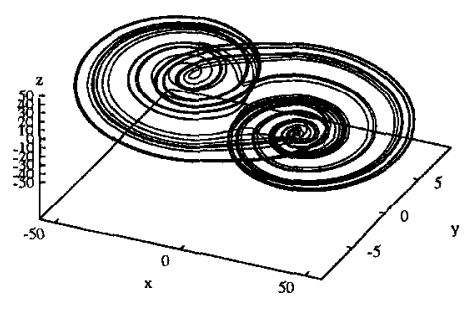

Fig. 2.21

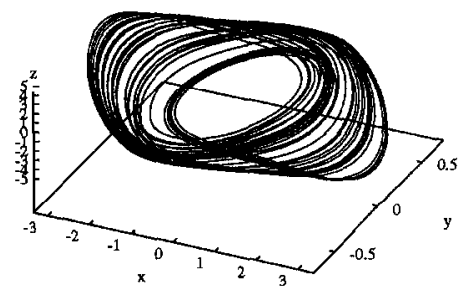

Fig. 2.22

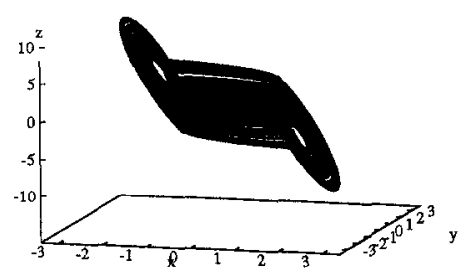

Fig. 2.23

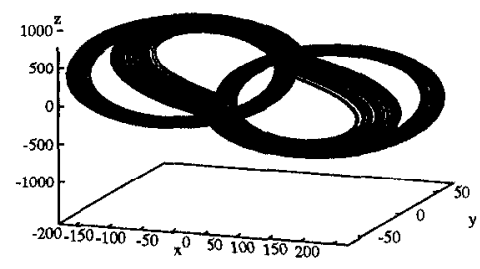

Fig. 2.24

FIG. 2-continued.

obtain a toroidal attractor. At the third Andronov-Hopf bifurcation, chaos is likely to appear. Both the torus breakdown route to chaos and the period-doubling route to chaos can be conveniently interpreted and explained in terms of the characteristic multipliers of the corresponding Poincaré map (13). In (11) an example of this route from a physical Chua's oscillator is presented.

2.5.3. Intermittency route to chaos. Intermittency is the phenomenon where the signal is virtually periodic except for some irregular (unpredictable) bursts. In other words, we have intermittently periodic behavior and irregular aperiodic behavior (11). In this route to chaos, the system is first periodic, then becomes chaotic as it exhibits intermittency. In (11), intermittency due to a tangent bifurcation is observed from a physical Chua's oscillator. 
L. Pivka et al.
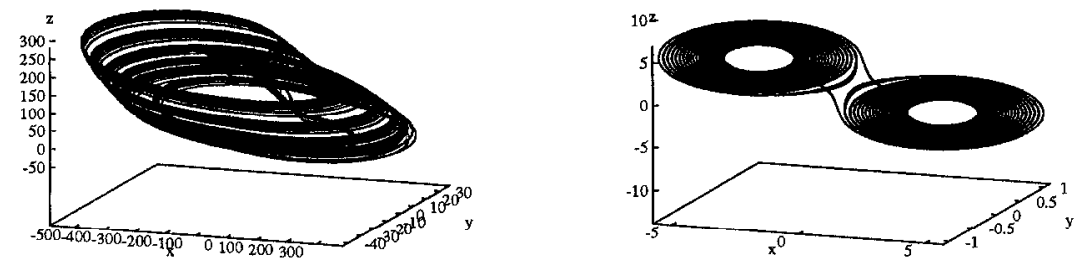

Fig. 2.25

Fig. 2.26
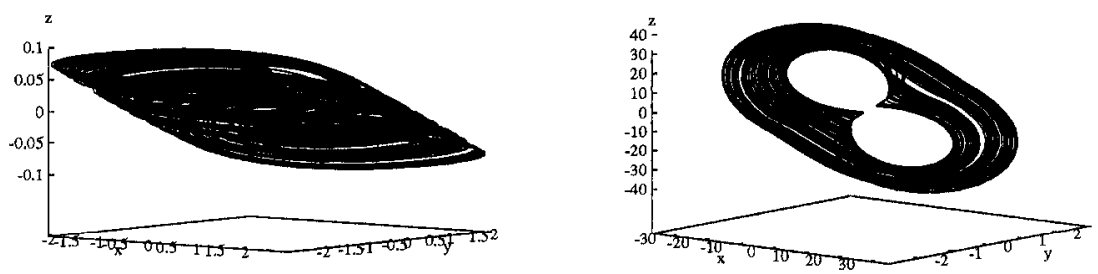

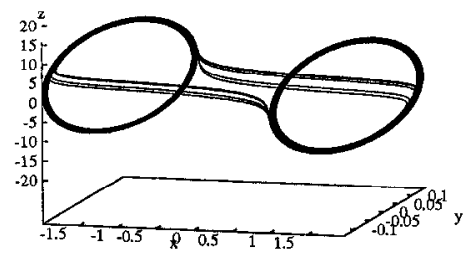

Fig. 2.29

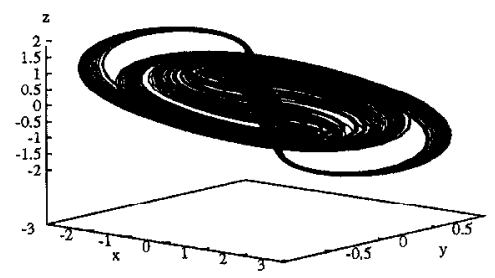

Fig. 2.30

FIG. 2-continued.

\subsection{Chaos-chaos intermittency and $1 / f$ noise}

It is known that interaction between chaotic attractors can give rise to intermittency - a random switching process between attractors after long periods of "laminar phases", when the trajectory stays near one of the attractors. A characteristic statistical property of the chaos-chaos type intermittency is the slope of its power spectrum in the low-frequency region. Such a property has also been observed (14) in Chua's circuit for parameter values near the birth of the Chua double scroll attractor. The power spectrum was numerically found to follow the law $S_{x}(w) \propto w^{-\delta}, \delta=1.1 \pm 0.1$, i.e. the graph on the double logarithmic scale clings to the ideal $1 / f$ line corresponding to $\delta=1$. The $1 / f$ spectrum has been observed previously in many processes of different origin, e.g. the fluctuations of the current 


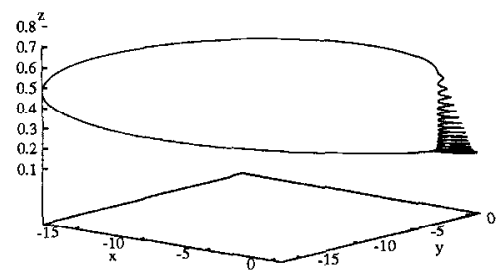

Fig. 2.31

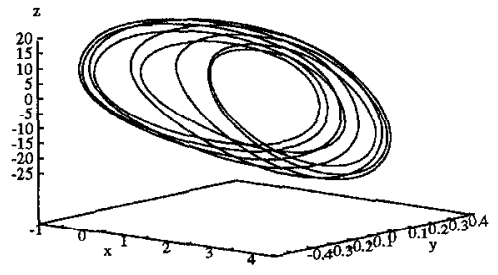

Fig. 2.32

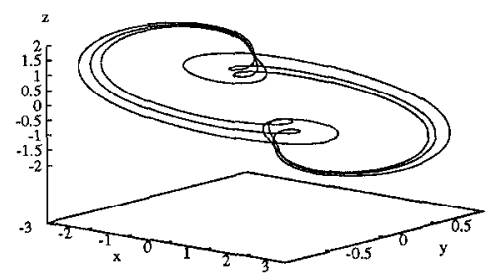

Fig. 2.33

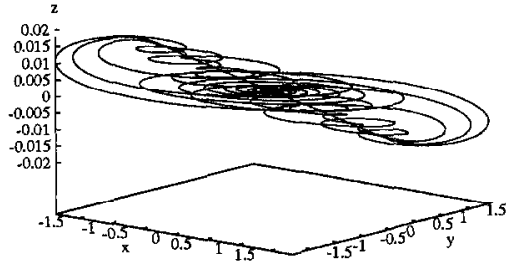

Fig. 2.34

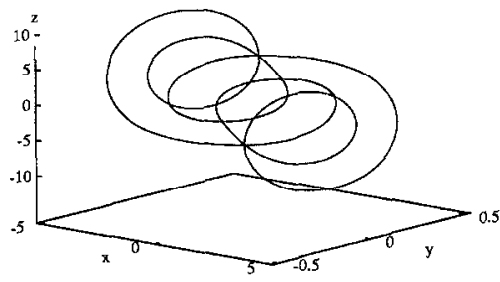

Fig. 2.35

FIG. 2-continued.

in electron devices, the fluctuations of the Earth's rotation frequency, the fluctuation of the muscle rhythms in the human heart, etc. and has been found to obey the above universal law. The intermittency phenomenon can be used as a $1 / f$ noise generator and can lead to a belter understanding of the ubiquitous yet still poorly understood $1 / f$ phenomenon.

\subsection{Stochastic resonance from Chua's circuit}

The phenomenon of stochastic resonance (SR) is observed in bistable nonlinear systems driven simultaneously by an external noise and a sinusoidal force. In this case, the signal-to-noise ratio (SNR) increases until it reaches a maximum at some optimum noise intensity $D$ which depends on the bistable system and on the 


\section{Pivka et al.}
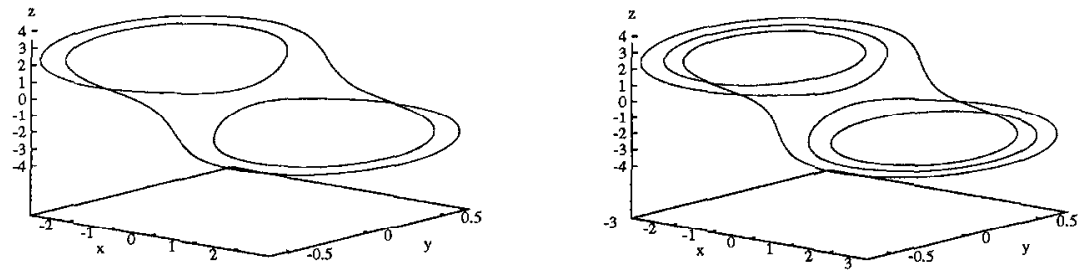

Fig. 3.1

Fig. 3.2
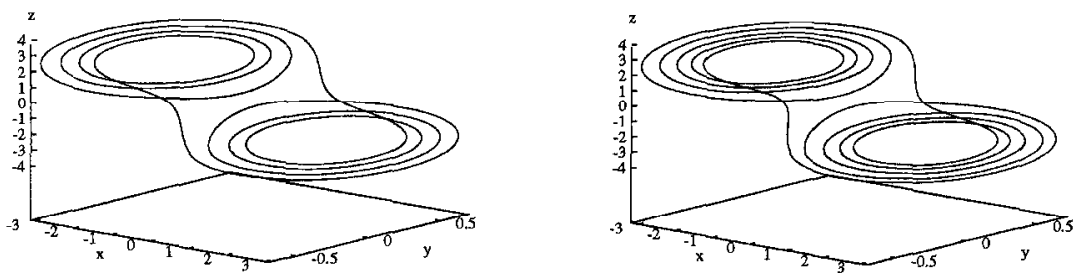

Fig. 3.4

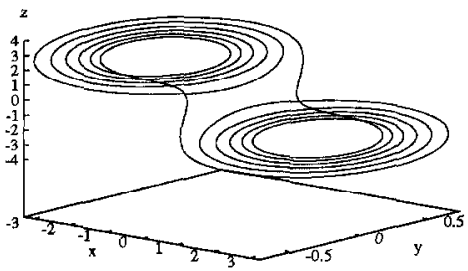

Fig. 3.5

FIG. 3. Attractors from a period-adding bifurcation sequence.

frequency of the external sinusoidal force. In the absence of a periodic modulation signal, the noise alone results in a random transition between the two states. This random process can be characterized by the mean switching frequency $w_{s}$, depending on the noise intensily $D$ and the height of the potential barrier separating the two stable states. In the presence of an external modulation imposed by the sinusoidal signal $A \sin (w t)$, the potential barrier changes periodically with time. 'The modulation signal amplitude $A$ is assumed to be sufficiently small so that the input signal alone does not induce transitions in the absence of noise. A coherence between the modulation frequency $w$ and the mean switching frequency $w_{s}$ emerges when the system is simultaneously driven by a periodic signal and a noise source. As a result, a part of the noise energy is transformed into the energy of the periodic 


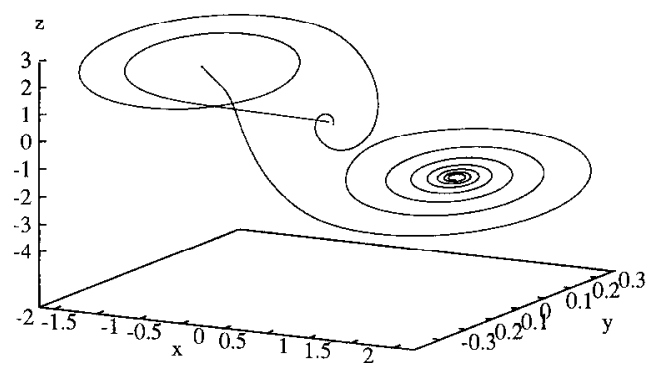

FIG. 4. Coexistence of homoclinic and heteroclinic orbits. The fixed parameters are $\alpha=8.29203, \beta=12.061126, \gamma=0, a=-1.1428571, b=-0.7142857, k=1$. Initial conditions: $x-1.519009798388814, y=-0.004298524877956949, z=-1.567578825979665$ (heteroclinic orbit) $; x=0.00083672759, y=0.000095112528, z=-0.00053929635$ (homoclinic orbit).

modulation signal so that the SNR increases. This phenomenon is qualitatively similar to the classical resonance phenomenon. However, unlike the classical circuit theory where one tunes the input frequency $w$ to achieve resonance in an RLC circuit, here $w$ is fixed at some convenient value and one tunes the noise intensity $D$ to achieve $\mathrm{SR}$.

In Chua's circuit, the SR phenomenon can be observed (15) in conjunction with the chaos-chaos type intermittency (14) arising in a small vicinity of the bifurcation curve in the $\alpha-\beta$ parameter space when two spiral attractors merge to form the double scroll attractor. In this case, the SNR of the amplified output signal is observed to be significantly greater than the SNR of the input signal-a novel phenomenon which cannot be achieved with a linear amplifier.

\subsection{Signal amplification via chaos}

Apart from the stochastic resonance phenomenon described above, another mechanism for achieving voltage gain (up to $50 \mathrm{~dB}$ has been demonstrated experimentally) from Chua's circuit has been discovered recently (16).

The mechanism of this voltage gain is different from that of stochastic resonance because the effect is observed even when Chua's circuit is operating in a spiral Chua's attractor regime far from the bifurcation boundary where stochastic resonance takes place.

\subsection{Chua's circuit with smooth nonlinearity}

Most of the studies on Chua's circuit and Chua's oscillator assume a piecewiselinear nonlinearity, although an arbitrary nonlinearity can be used. Since the characteristics of nonlinear resistors in real circuits are always smooth, a question arises as to whether the phenomena in piecewise-linear and smooth models coincide: This question is approached in (17) by demonstrating that most phenomena from the piecewise-linear Chua's circuit (e.g. the double scroll) carry over to the smooth model with a cubic polynomial for the nonlinear function. Also most of the bifurcations (period-doubling, for instance) in the smooth model appear to be similar to those in the piecewise-linear model (see, for example, (18) which also 


\section{Pivka et al.}

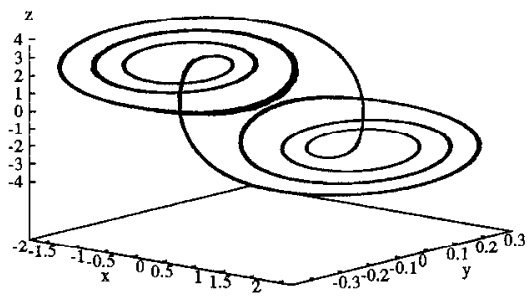

Fig. 5.1

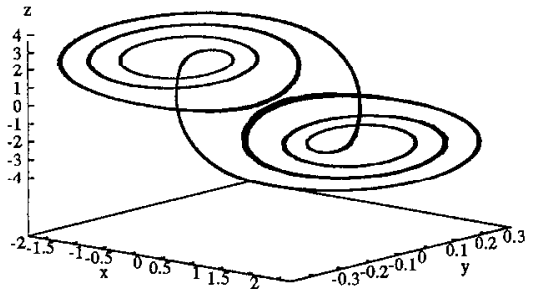

Fig. 5.2

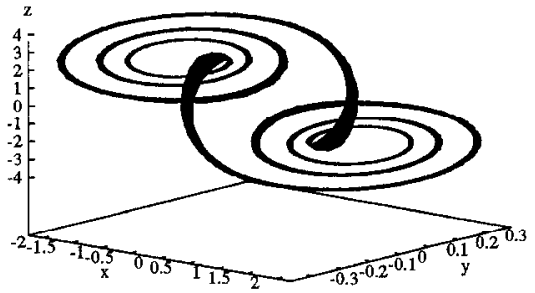

Fig. 5.3

FIG. 5. Coexistence of three chaotic attractors. The fixed parameters are $\alpha=15.6$, $\beta=28.58000012, \gamma=0, a=-1.14285714, b=-0.7142857, k=1$. Initial conditions: $x=-1.955798, \quad y=-0.2269574, \quad z=-1.85494 \quad$ (attractor 1$) ; \quad x=1.955798$, $y=0.2269574, z=1.85494$ (attractor -1 ) $; x=0.65153, y=0.10764, z=-1.5407$ (oddsymmetric attractor 2). 


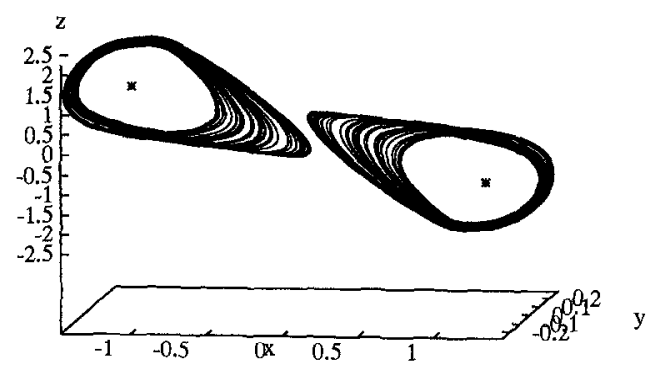

Fig. 6.1

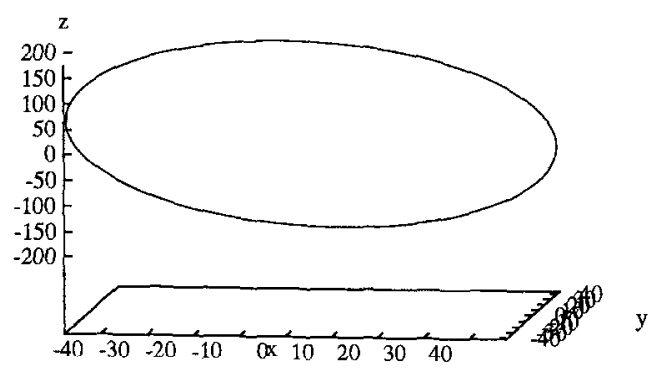

Fig. 6.2

Fig. 6. Coexistence of two point attractors, two chaotic attractors (Fig. 6.1), and a periodic attractor (Fig. 6.2). The fixed parameters are $\alpha=9.50028501, \beta=14.2857, \gamma=0$, $a=-1.142856, b=-0.142857, k=1$.

gives an implementation of a cubic polynomial $v-i$ characteristic using analog multipliers).

2.10. Self-similar and universal structures in two-parameter study of transition to chaos

Using the Poincaré map technique, the exact description of the system [Eq. (1)] can be reduced to a two-dimensional map which, in turn, can be approximated by a one-dimensional map (19) generally called Chua's 1D map in the literature. Such an approximation is possible because of the strong dissipation of the system which "flattens out" the dynamics. This map happens to be bimodal in certain parameter regions, which means that it has both a maximum and a minimum on an interval which is mapped onto itself. The condition is responsible for the 


\section{Pivka et al.}

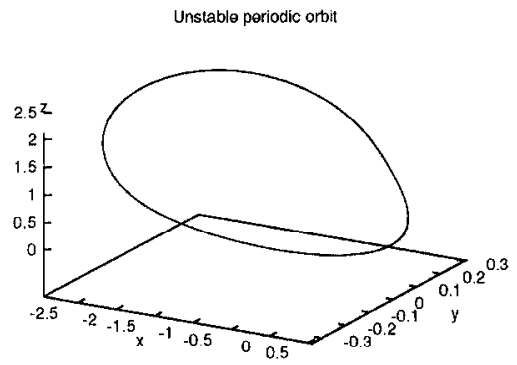

Fig. 7.1

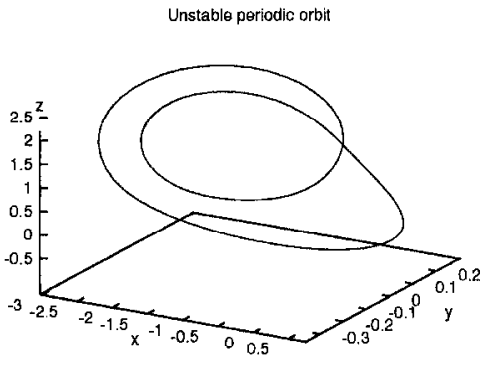

Fig. 7.2

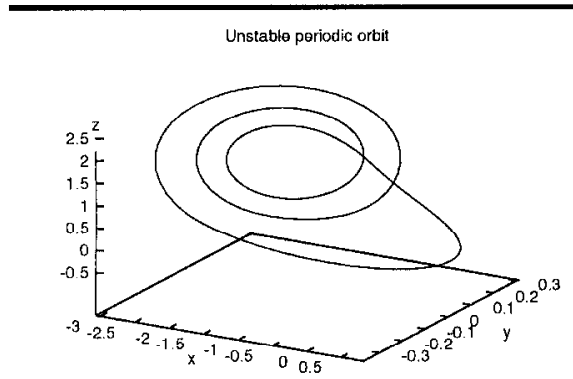

Fig. 7.3

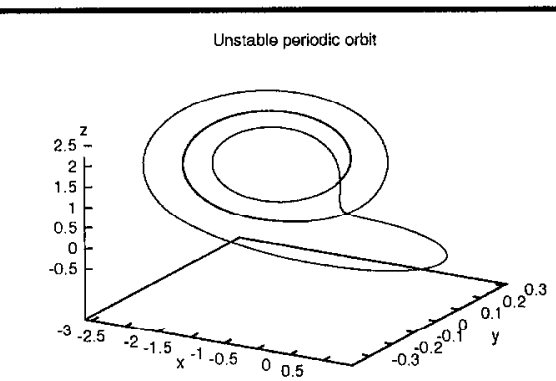

Fig. 7.5

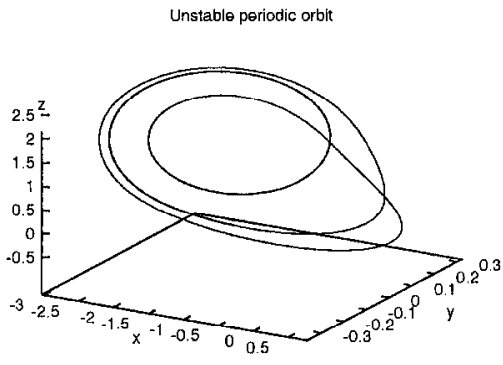

Fig. 7.4

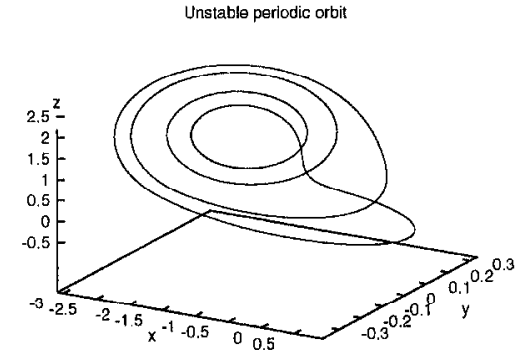

Fig. 7.6

FIG. 7. Unstable periodic orbits. In Fig. 7.13 the unstable periodic orbits are shown superimposed; in Fig. 7.14 the coexisting double scroll Chua's attractor is shown. The fixed parameters are $\alpha=9, \beta=100 / 7, \gamma=0, a=-8 / 7, b=-5 / 7, k=1$.

complicated structure of the boundary of chaos in a two-parameter bifurcation diagram.

In a typical one-parameter bifurcation sequence, if we tune only one parameter in Chua's circuit, we usually see a typical period-doubling cascade, which exhibits remarkable properties of quantitative universality (20) and self-similarity, namely, an interval encompassing regions of different dynamical regimes reproduces itself under a change in scale by the universal factor $\delta=4.6692 \ldots$ (see, for example, (21)). 


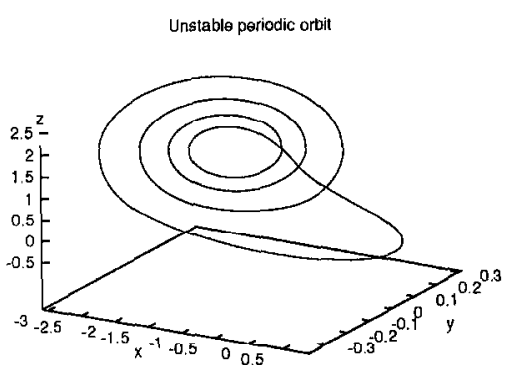

Fig. 7.7

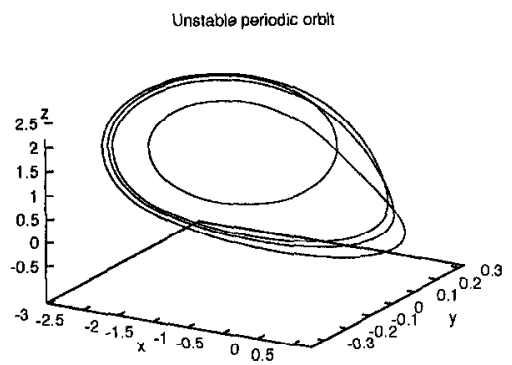

Fig. 7.8

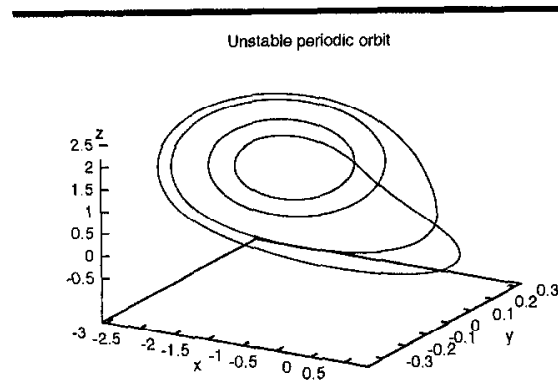

Fig. 7.9

Unstable periodic orbi

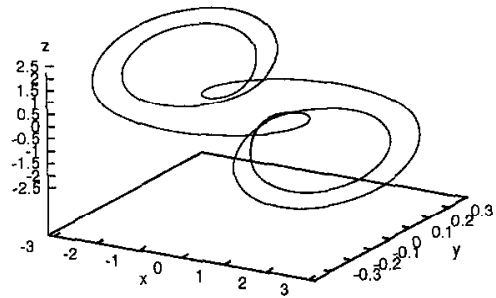

Fig. 7.11

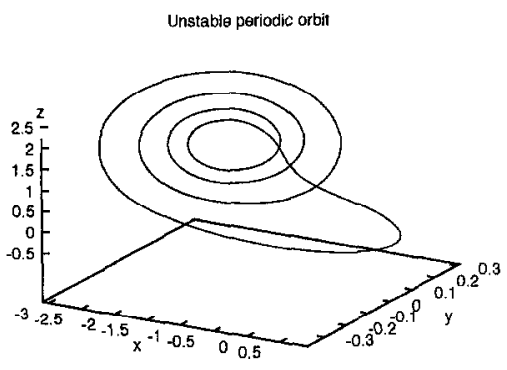

Fig. 7.10

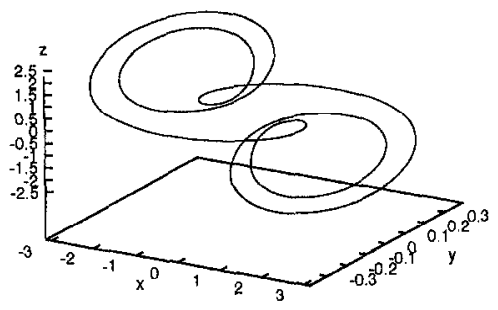

Fig. 7.12

FIG. 7-continued.

If we turn to a two-parameter study, we can no longer restrict ourselves to the Feigenbaum scenario which is a codimension- 1 bifurcation phenomenon. In (22) the construction of a binary tree of superstable orbits is performed for the $1 \mathrm{D}$ Chua's map to show that beside the Feigenbaum critical lines, the boundary of chaos contains an infinite number of codimension- 2 critical points, defined by a set of infinite binary codes. The topography of the parameter plane near the corresponding critical points reveals a property of two-parameter self-similarity: a two-dimensional structure of regions of different behavior is reproduced under a 


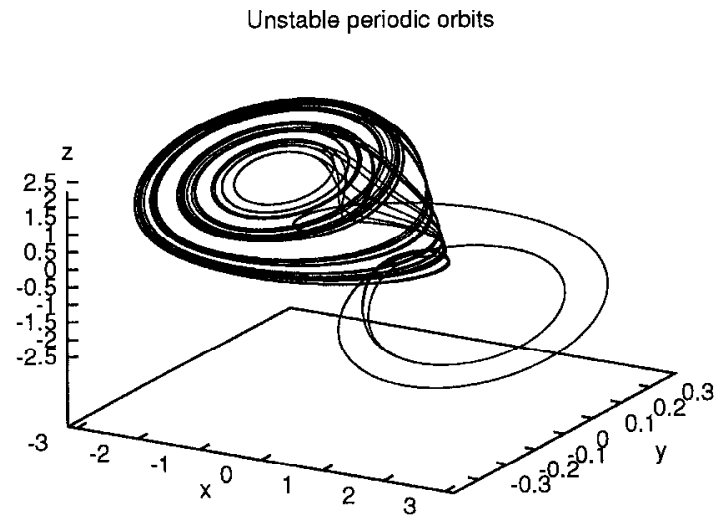

Fig. 7.13

Double Scroll Chua's attractor

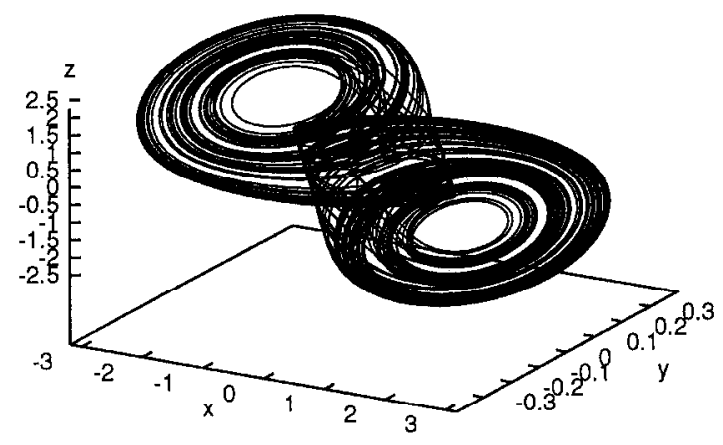

Fig. 7.14

FIG. 7-continued.

scale change along appropriate axes in the parameter space. These self-similar twodimensional patterns are universal (up to a linear parameter change) for all bimodal maps, and depend only on the code of the associated critical point. Moreover, two universal scaling numbers have been found for the two-parameter 1D maps, which are a generalization of the Feigenbaum number.

\subsection{Antimonotonicity phenomenon}

Antimonotonicity - concurrent creation and annihilation of periodic orbits, or inevitable reversals of period-doubling cascades - was shown to be a fundamental phenomenon for a large class of nonlinear systems (23). Experimental (24) and numerical (10) evidence was given that this phenomenon is typical for a wide range of parameters in Chua's circuit and Chua's oscillator, respectively. 


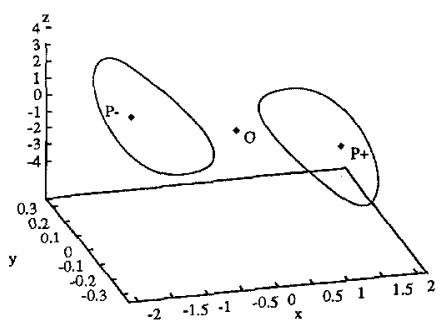

Fig. 8.1

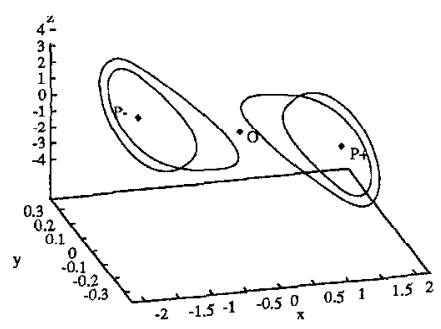

Fig. 8.2

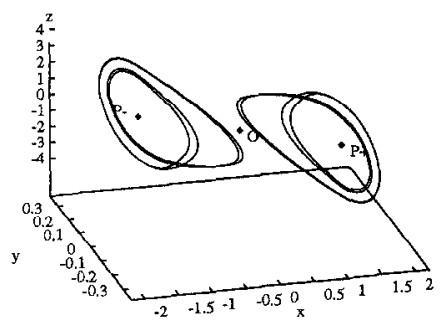

Fig. 8.3

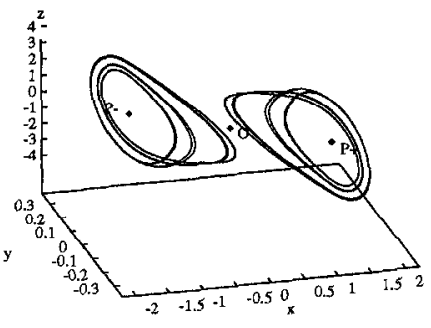

Fig. 8.4

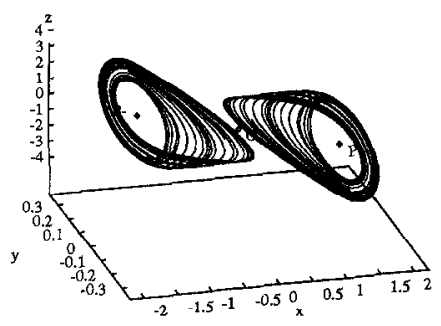

Fig. 8.5

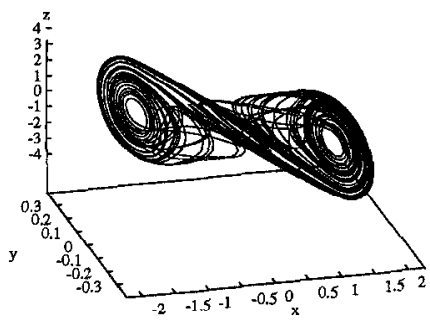

Fig. 8.6

FIG. 8. Period-doubling route to chaos. The fixed parameters are $\beta=16, \gamma=0, a=-8 / 7$, $b=-5 / 7 ; k=1 ; \alpha=8.8$ (period-1), $\alpha=9.05$ (period-2), $\alpha=9.12$ (period- 4$), \alpha=9.162$ (period-8), $\alpha=9.3$ (spiral attractor), $\alpha=9.8$ (double scroll attractor).

\subsection{Devil's staircase from the driven Chua's circuit}

One of the remarkable properties of nonlinear oscillators is their ability to lock onto certain subharmonic frequencies when driven by an external source of energy. Associated with the phase-locking property is usually the appearance of "staircases" of phase-locked states when the parameters are varied over a certain range. The picturesque name devil's staircase is used to describe the intricate, often fractal, structure of such staircases. Figure 9 shows the devil's staircase in Chua's circuit obtained by plotting the ratio of winding numbers and period numbers as a function of the normalized forcing angular frequency (see, for example (25)). The 


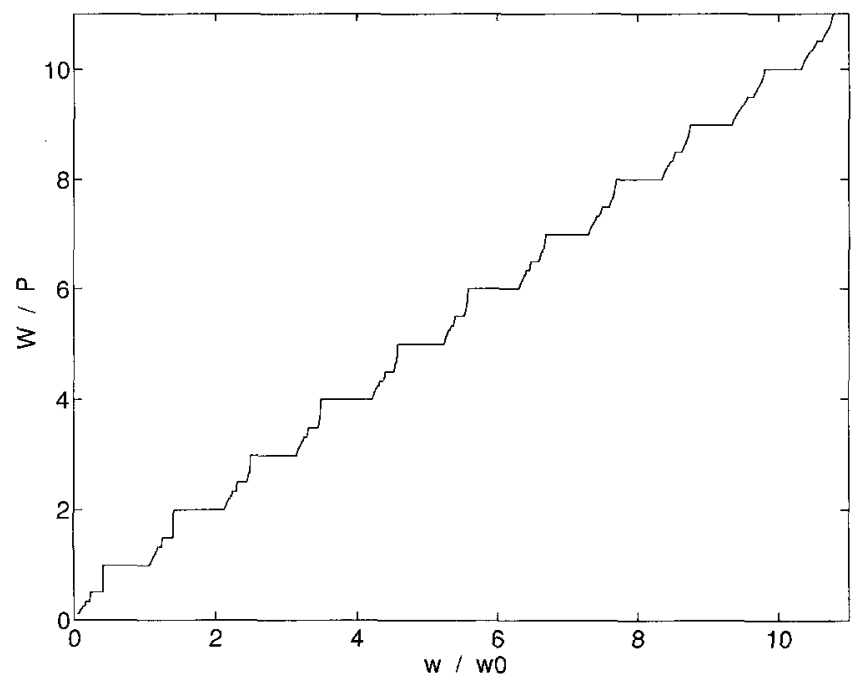

FIG. 9. Devil's staircase from the sinusoidally driven Chua's circuit.

self-similar structure of the staircase tree and the devil's staircase becomes apparent when magnified pictures are drawn of the portions of the devil's staircase.

\subsection{Other dynamical phenomena from the driven Chua's oscillator}

Extensive computer simulations and physical experiments were performed in a two-parameter study (26) to describe several types of transition to chaos in the nonautonomous Chua's circuit. Also in an experimental and numerical study (27) of Chua's oscillator some new phenomena - frequency entrainment of chaos, period-preserving bifurcations have been reported, along with many other phenomena previously observed from different oscillators.

\section{Global Unfolding Theorem}

In this section, we show that Chua's oscillator is topologically conjugate (up to time scale) to a large class of piecewise-linear vector fields. The class of vector fields we are considering will be

- 3-dimensional,

- continuous,

- piecewise-linear with three regions separated by boundary planes, and

- odd-symmetric with respect to the origin.

We will call this class of vector fields $\mathscr{C}$. Without loss of generality, we can assume that the boundary planes are of the form $\mathbf{e}_{1}^{T} \mathbf{x}= \pm 1$, where $\mathbf{e}_{1}=(1,0,0)^{T}$. Then every member of the class $\mathscr{C}$ can be represented by 


$$
\dot{\mathbf{x}}= \begin{cases}\mathbf{A}_{1} \mathbf{x}+\mathbf{b}, & \mathbf{e}_{1}^{T} \mathbf{x} \geqslant 1 \\ \mathbf{A}_{0} \mathbf{x}, & -1 \leqslant \mathbf{e}_{1}^{T} \mathbf{x} \leqslant 1 \\ \mathbf{A}_{1} \mathbf{x}-\mathbf{b}, & \mathbf{e}_{1}^{T} \mathbf{x} \leqslant-1 .\end{cases}
$$

By continuity, we must have

$$
\mathbf{A}_{0}=\mathbf{A}_{1}+\mathbf{b e}_{1}^{T} \text {. }
$$

Thus class $\mathscr{C}$ is a 12-parameter family of ordinary differential equations. The global unfolding theorem (8) tells us that (most of) this class is topologically equivalent to the Chua's oscillator which has six parameters. System (4) can be written as:

$$
\dot{\mathbf{x}}=\mathbf{A}_{1} \mathbf{x}+\frac{1}{2}\left(\left|\mathbf{e}_{1}^{T} \mathbf{x}+1\right|-\left|\mathbf{e}_{1}^{T} \mathbf{x}-1\right|\right) \mathbf{b} .
$$

Let us denote the characteristic polynomials of $\mathbf{A}_{0}$ and $\mathbf{A}_{1}$ as follows :

$$
\begin{aligned}
& \chi\left(\mathbf{A}_{0}\right)=\lambda^{3}-p_{1} \lambda^{2}+p_{2} \lambda-p_{3} \\
& \chi\left(\mathbf{A}_{1}\right)=\lambda^{3}-q_{1} \lambda^{2}+q_{2} \lambda-q_{3} .
\end{aligned}
$$

If $\left(\mu_{1}, \mu_{2}, \mu_{3}\right)$ are the eigenvalues of $\mathbf{A}_{0}$ and $\left(v_{1}, v_{2}, v_{3}\right)$ are the eigenvalues of $\mathbf{A}_{1}$, then the coefficients of the characteristic polynomials are expressed as

$$
\begin{array}{ll}
p_{1}=\mu_{1}+\mu_{2}+\mu_{3} & q_{1}=v_{1}+v_{2}+v_{3} \\
p_{2}=\mu_{1} \mu_{2}+\mu_{2} \mu_{3}+\mu_{3} \mu_{1} & q_{2}=v_{1} v_{2}+v_{2} v_{3}+v_{3} v_{1} \\
p_{3}=\mu_{1} \mu_{2} \mu_{3}, & q_{3}=v_{1} v_{2} v_{3} .
\end{array}
$$

Theorem 1 (Global Unfolding Theorem)

Consider system (4). Let the entries of $\mathbf{A}_{1}$ be denoted as

$$
\mathbf{A}_{1}=\left(\begin{array}{lll}
a_{11} & a_{12} & a_{13} \\
a_{21} & a_{22} & a_{23} \\
a_{31} & a_{32} & a_{33}
\end{array}\right) .
$$

Suppose that $\mathbf{A}_{1}$ satisfies the inequality

$$
a_{12} a_{12} a_{23}+a_{12} a_{13} a_{33}-a_{12} a_{13} a_{22}-a_{13} a_{13} a_{32} \neq 0 \text {. }
$$

Let us define

$$
\begin{aligned}
& k_{1} \triangleq-p_{3}+\left(\begin{array}{l}
q_{3}-p_{3} \\
q_{1}-p_{1}
\end{array}\right)\left(p_{1}+\frac{p_{2}-q_{2}}{q_{1}-p_{1}}\right) \\
& k_{2} \triangleq p_{2}-\left(\frac{q_{3}-p_{3}}{q_{1}-p_{1}}\right)+\left(\frac{p_{2}-q_{2}}{q_{1}-p_{1}}\right)\left(\frac{p_{2}-q_{2}}{q_{1}-p_{1}}+p_{1}\right) \\
& k_{3} \triangleq\left(\frac{p_{2}-q_{2}}{q_{1}-p_{1}}\right)-\frac{k_{1}}{k_{2}} \\
& k_{4} \triangleq-k_{1} k_{3}+k_{2}\left(\frac{p_{3}-q_{3}}{p_{1}-q_{1}}\right) .
\end{aligned}
$$




\section{Pivka et al.}

If the coefficients of the characteristic polynomials of $\mathbf{A}_{0}$ and $\mathbf{A}_{1}$ satisfy the inequalities

$$
\begin{aligned}
p_{1}-q_{1} & \neq 0 \\
k_{2} & \neq 0 \\
k_{3} & \neq 0 \\
k_{4} & \neq 0
\end{aligned}
$$

then the vector field in the form (4) is topologically equivalent to a Chua's oscillator with the parameters

$$
\begin{aligned}
& x=-\frac{k_{2}}{k_{3}^{2}} \\
& \beta=\frac{k_{4}}{k_{2} k_{3}^{2}} \\
& \gamma=\frac{k_{1}}{k_{2} k_{3}} \\
& a=-1+\left(p_{1}+\frac{-p_{2}+q_{2}}{p_{1}-q_{1}}\right) \frac{k_{3}}{k_{2}} \\
& b=-1+\left(q_{1}+\frac{-p_{2}+q_{2}}{p_{1}-q_{1}}\right) \frac{k_{3}}{k_{2}} \\
& k=\operatorname{sgn}\left(k_{3}\right) .
\end{aligned}
$$

Proof: Define the matrix $\mathbf{K}$ as follows :

$$
\mathbf{K}=\left(\begin{array}{ccc}
1 & 0 & 0 \\
a_{11} & a_{12} & a_{13} \\
\sum_{j=1}^{3} a_{1 j} a_{j 1} & \sum_{j=1}^{3} a_{1 j} a_{j 2} & \sum_{j=1}^{3} a_{1, j} a_{j 3}
\end{array}\right)
$$

By the hypothesis, $\mathbf{K}$ is nonsingular. Using the transformation $\mathbf{y}=\mathbf{K} \mathbf{x}$, we obtain a system of the form

$$
\dot{\mathbf{y}}=\mathbf{K A} \mathbf{A}_{l} \mathbf{K}^{-1} \mathbf{y}+\frac{1}{2}\left(\left|\mathbf{e}_{1}^{T} \mathbf{K}^{-1} \mathbf{y}+1\right|-\left|\mathbf{e}_{1}^{T} \mathbf{K}^{-1} \mathbf{y}-1\right|\right) \mathbf{K} \mathbf{b} .
$$

It is clear from the definition of $\mathbf{K}$ that the first row of $\mathbf{K}^{-1}$ is of the form $(1,0,0)$. This implies that $\mathbf{e}_{1}^{T} \mathbf{K}^{-1}=\mathbf{e}_{1}^{T}$. After some algebraic manipulation, it can be shown that

$$
\mathbf{K} \boldsymbol{\Lambda}_{1} \mathbf{K}^{-1}=\left(\begin{array}{ccc}
0 & 1 & 0 \\
0 & 0 & 1 \\
q_{3} & -q_{2} & q_{1}
\end{array}\right)
$$


Now $\mathbf{K} \mathbf{A}_{0} \mathbf{K}^{-1}=\mathbf{K}\left(\mathbf{A}_{1}+\mathbf{b} \mathbf{e}_{1}^{T}\right) \mathbf{K}^{-1}=\mathbf{K} \mathbf{A}_{1} \mathbf{K}^{-1}+\mathbf{K b} \mathbf{e}_{1}^{T} . \quad$ If we write $\mathbf{K b}=$ $\left(\tilde{b}_{1}, \tilde{b}_{2}, \tilde{b}_{3}\right)^{T}$, then

$$
\mathbf{K} \mathbf{A}_{0} \mathbf{K}^{-1}=\left(\begin{array}{ccc}
\tilde{b}_{1} & 1 & 0 \\
\tilde{b}_{2} & 0 & 1 \\
\tilde{b}_{3}+q_{3} & -q_{2} & q_{1}
\end{array}\right) \text {. }
$$

Calculating the characteristic polynomial of $\mathbf{K A}_{0} \mathbf{K}^{-1}$ and comparing it with Eq. (6), we obtain

$$
\left(\begin{array}{ccc}
1 & 0 & 0 \\
-q_{1} & 1 & 0 \\
q_{2} & -q_{1} & 1
\end{array}\right)\left(\begin{array}{l}
\tilde{b}_{1} \\
\tilde{b}_{2} \\
\tilde{b}_{3}
\end{array}\right)=\left(\begin{array}{c}
p_{1}-q_{1} \\
q_{2}-p_{2} \\
p_{3}-q_{3}
\end{array}\right)
$$

Thus $\mathbf{K b}$ is uniquely determined by the parameters $\left(p_{1}, p_{2}, p_{3}, q_{1}, q_{2}, q_{3}\right)$.

In particular, we obtain the following equivalent form for system (4) :

$$
\begin{aligned}
\dot{\mathbf{y}} & =\left(\begin{array}{ccc}
0 & 1 & 0 \\
0 & 0 & 1 \\
q_{3} & -q_{2} & q_{1}
\end{array}\right) \mathbf{y} \\
& +\frac{1}{2}\left(\left|\mathbf{e}_{1}^{T} \mathbf{y}+1\right|-\left|\mathbf{e}_{1}^{T} \mathbf{y}-1\right|\right)\left(\begin{array}{c}
p_{1}-q_{1} \\
p_{3}-q_{3}-q_{2}\left(p_{1}-q_{1}\right)+q_{1}\left(-p_{2}+q_{2}+q_{1}\left(p_{1}-q_{1}\right)\right)
\end{array}\right)
\end{aligned}
$$

which is uniquely determined by the eigenvalues of $\mathbf{A}_{0}$ and $\mathbf{A}_{1}$.

When Chua's oscillator is written in the form of Eq. (4), we get

$$
\mathbf{A}_{0}=\left(\begin{array}{ccc}
-k \alpha(1+a) & k \alpha & 0 \\
k & -k & k \\
0 & -k \beta & -k \gamma
\end{array}\right), \quad \mathbf{A}_{1}=\left(\begin{array}{ccc}
-k \alpha(1+b) & k \alpha & 0 \\
k & -k & k \\
0 & -k \beta & -k \gamma
\end{array}\right) .
$$

It can be shown after some involved algebraic manipulation that if the inequalities (10)-(13) are satisfied and the parameters satisfy Eq. (14), then the eigenvalues of $\mathbf{A}_{0}$ and $\mathbf{A}_{1}$ in Eq. (17) satisfy Eq. (6), up to a positive scale factor. Given these parameters, it is elcar that the matrix $\mathbf{K}$ corresponding to Chua's oscillator is nonsingular, so we can also write the state equations of Chua's oscillator in the form of Eq. (16) $\dagger$. Thus we have shown that given the conditions in the theorem, both Chua's oscillator and a vector field of the form of Eq. (4) can be transformed into the same form, and are thus topologically equivalent.

This theorem can be summarized as the following algorithm :

1. Calculate the coefficients $\left(p_{1}, p_{2}, p_{3}, q_{1}, q_{2}, q_{3}\right)$ of the characteristic polynomials of $\mathbf{A}_{0}$ and $\mathbf{A}_{1}$.

$\dagger$ After renormalization of time. 


\section{Pivka et al.}

2. Check whether the inequalities (9), and (10)-(13) are satisfied.

3. Calculate the parameters of Chua's oscillator using Eq. (14).

In Step 2 of the algorithm, if the inequalities are not satisfied, in general it is possible to satisfy these inequalities by perturbing the entries of $\mathbf{A}_{0}$ or $\mathbf{A}_{1}$ slightly to get a system with similar behavior.

\section{Applications of the Global Unfolding Theorem}

Because of the generality of Chua's oscillator, other chaotic systems can be modelled using Chua's oscillator. The reader is referred to $(\mathbf{8}, \mathbf{2 8})$ for several examples of circuits and systems belonging to the class $\mathscr{C}$ of vector fields defined above which have been transformed into a "qualitatively similar" Chua's oscillator. These examples include the systems studied in (29-32). In this section we will illustrate this procedure with several additional examples.

In the following examples, the system under consideration is either already a 3 dimensional piecewise-linear three-segment continuous odd-symmetric vector field where the partition planes are parallel, or else can be approximated by one. When the vector field is not piecewise-linear, we approximate it by calculating the Jacobian matrices at the equilibrium points and using them to define the linear vector field in each region.

We then find the eigenvalues in each linear region and apply the above algorithm to find the parameters for Chua's oscillator. For cases where the vector field does not satisfy the inequalities in Step 2 of the algorithm, we perturb the eigenvalues (or equivalent eigenvalue parameters) slightly.

\subsection{Example from Arnéodo et al.}

The systems studied in (33-35) satisfy the following differential equation:

$$
\ddot{A}+\mu_{2} \ddot{A}+\mu_{1} \dot{A}+\mu_{0} A= \pm A^{3} .
$$

In (34), the cubic nonlinearity is replaced by a three-segment piecewise-linear nonlinearity resulting in a vector field in $\mathscr{C}$. We have two cases, depending on whether the right hand side is $+A^{3}$ or $-A^{3}$.

Case 1 (right hand side is $+A^{3}$ ):

$$
\left.\begin{array}{l}
\dot{x}=y \\
\dot{y}=z \\
\dot{z}=x^{3}-\mu_{0} x-\mu_{1} y-\mu_{2} z .
\end{array}\right\}
$$

The equilibrium points are as follows: $\left(\sqrt{\mu_{0}}, 0,0\right),(0,0,0)$, and $\left(-\sqrt{\mu_{0}}, 0,0\right)$. From Eq. (19), the Jacobian matrix is

$$
M=\left[\begin{array}{ccc}
0 & 1 & 0 \\
0 & 0 & 1 \\
3 x^{2}-\mu_{0} & -\mu_{1} & -\mu_{2}
\end{array}\right]
$$


We choose $\mu_{0}=9.6, \mu_{1}=5$, and $\mu_{2}=1$; the Jacobian matrix at the equilibrium points in the two outside regions is

$$
M=\left[\begin{array}{ccc}
0 & 1 & 0 \\
0 & 0 & 1 \\
19.2 & -5 & -1
\end{array}\right]
$$

In the inner region the Jacobian matrix at the equilibrium point is

$$
M_{0}=\left[\begin{array}{ccc}
0 & 1 & 0 \\
0 & 0 & 1 \\
-9.6 & -5 & -1
\end{array}\right]
$$

As the Jacobian matrix is already in companion form, the corresponding equivalent eigenvalue parameters can be read off directly:

$$
\left.\begin{array}{ccc}
p_{1}=-1.0, & p_{2}=5, \quad p_{3}=-9.6 \\
q_{1}=-1.0, \quad q_{2}=5, & q_{3}=19.2 .
\end{array}\right\}
$$

Since $p_{1}=q_{1}$ (i.e. the inequality (10) in Step 2 of the algorithm in the preceding section is not satisfied), we add a small perturbation $\delta p_{1}=0.05$, and $\delta q_{1}=-0.05$ to obtain

$$
\left.\begin{array}{c}
p_{1}^{\prime}=-0.95, \quad p_{2}^{\prime}=5, \quad p_{3}^{\prime}=-9.6 \\
q_{1}^{\prime}=-1.05, \quad q_{2}^{\prime}=5, \quad q_{3}^{\prime}=19.2 .
\end{array}\right\}
$$

The corresponding dimensionless parameters are

$$
\left.\begin{array}{ccc}
\alpha=-313.6291, & \beta=-307.2771, & \gamma=-1, \\
a=-0.9968661, & b=-0.9965362, & \tau=-0.9665529, \\
k=-1 . &
\end{array}\right\}
$$

By using these parameters we obtain the attractor shown in Fig. 10.5(b) which is qualitatively similar to the attractor in Fig. 1(a) of (35).

Case 2 (right hand side is $-A^{3}$ ):

$$
\left.\begin{array}{l}
\dot{x}=y \\
\dot{y}=z \\
\dot{z}=-x^{3}-\mu_{0} x-\mu_{1} y-\mu_{2} z .
\end{array}\right\}
$$

The equilibrium points are as follows: $\left(\sqrt{-\mu_{0}}, 0,0\right),(0,0,0)$, and $\left(-\sqrt{-\mu_{0}}, 0,0\right)$

From Eq. (23), the Jacobian matrix is

$$
M=\left[\begin{array}{ccc}
0 & 1 & 0 \\
0 & 0 & 1 \\
-3 x^{2}-\mu_{0} & -\mu_{1} & -\mu_{2}
\end{array}\right]
$$




\section{Pivka et al.}

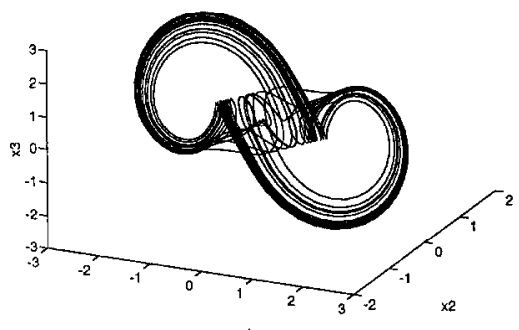

Fig. 10.1a

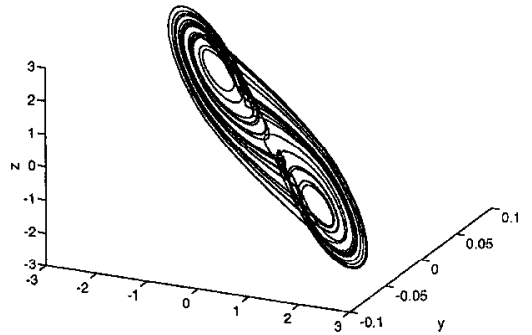

$x$

Fig. $10.1 \mathrm{~b}$

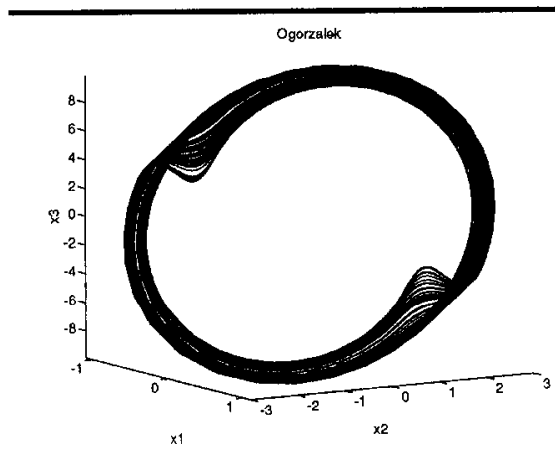

Fig. 10.2a

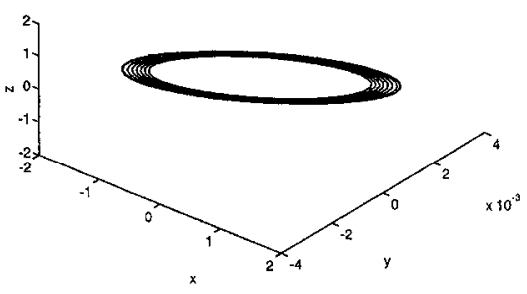

Fig. 10.2b

\section{Sparrow}

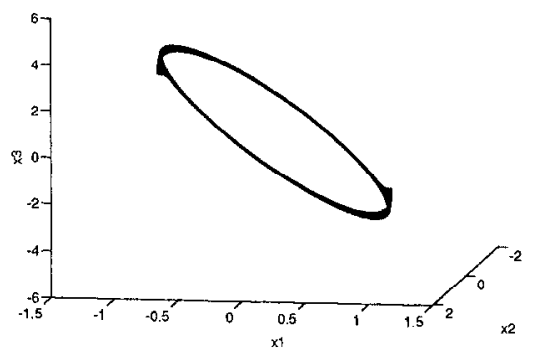

Fig. 10.3a

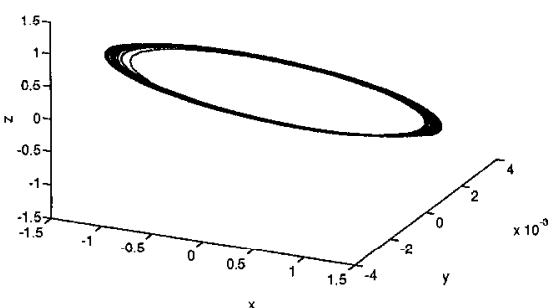

Fig. $10.3 \mathrm{~b}$

FIG. 10. Chaotic attractors from different systems, mimicked by Chua's oscillator. On the left (Fig. 10.1a, 10.2a, etc.) we show an attractor from the original system, while on the right (Fig. $10.1 \mathrm{~b}, 10.2 \mathrm{~b}$, ctc.) we show the corresponding attractor from Chua's oscillator. The parameters are shown in Table II. 


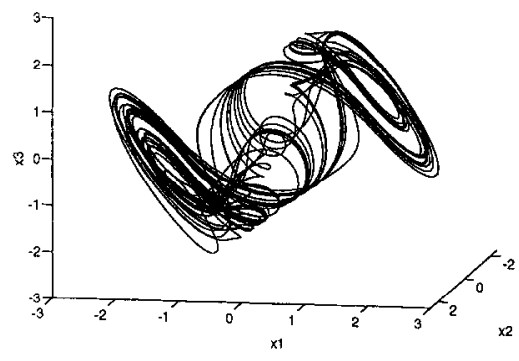

Fig. 10.4a

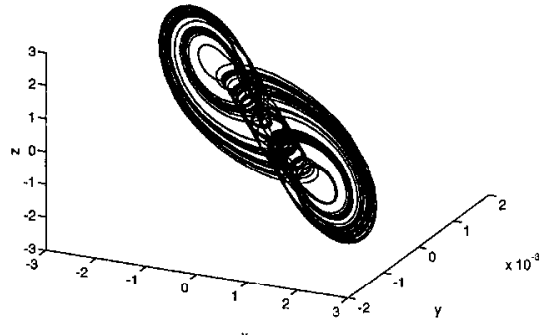

Fig. $10.4 \mathrm{~b}$

Arneodo1

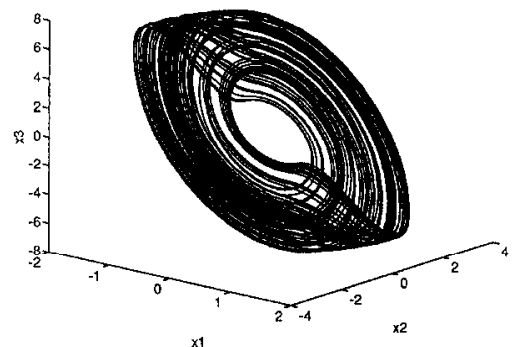

Fig. 10.5a

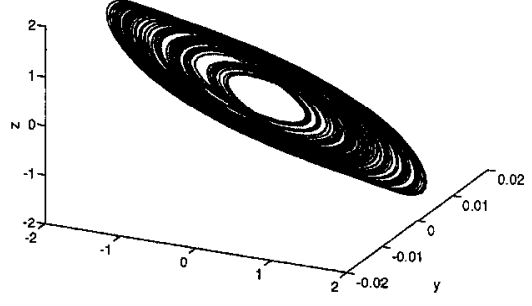

Fig. 10.5b

Arneoda2

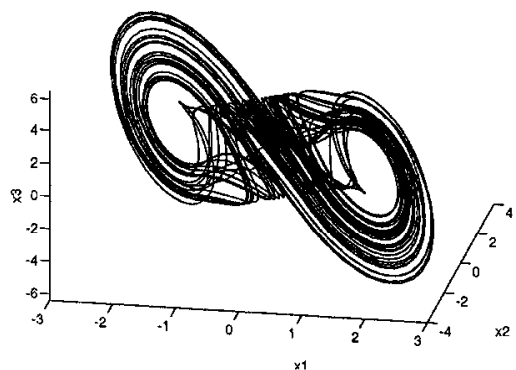

Fig. 10.6a

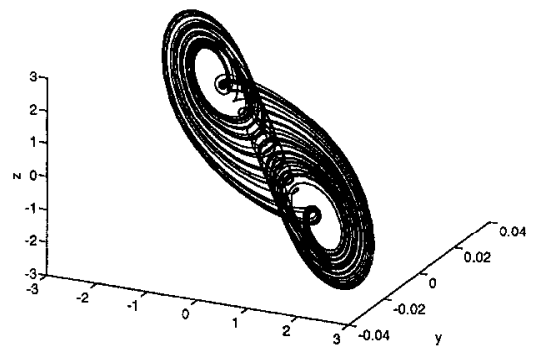

Fig. $10.6 \mathrm{~b}$

FIG. 10-continued. 


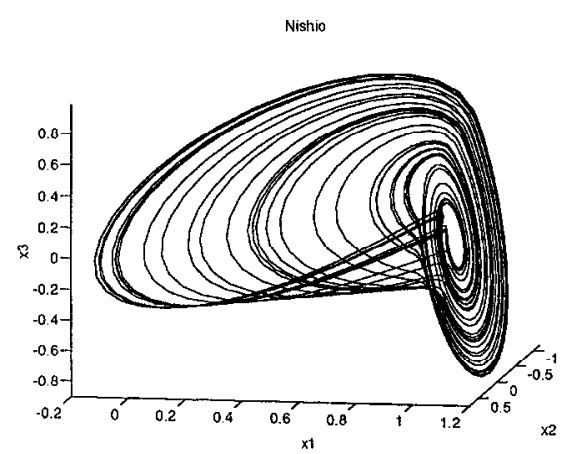

Fig. 10.7a

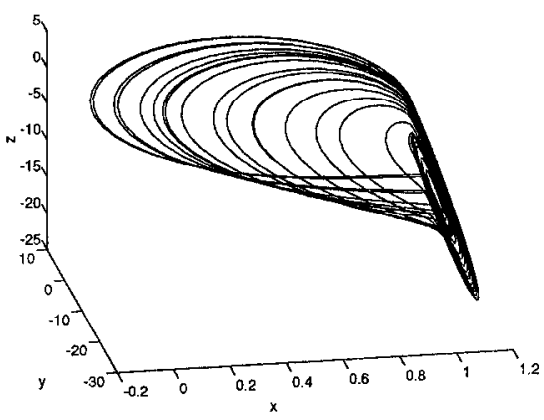

Fig. $10.7 \mathrm{~b}$

FIG. 10 continued.

We choose $\mu_{0}=-5.5, \mu_{1}=3.5$, and $\mu_{2}=1.1$, and in the two outside regions the Jacobian matrix at the equilibrium points is

$$
M=\left[\begin{array}{ccc}
0 & 1 & 0 \\
0 & 0 & 1 \\
-11 & -3.5 & -1.1
\end{array}\right]
$$

In the inner region, the Jacobian matrix at the equilibrium point is

$$
M_{0}=\left[\begin{array}{ccc}
0 & 1 & 0 \\
0 & 0 & 1 \\
5.5 & -3.5 & -1.1
\end{array}\right] \text {. }
$$

The equivalent eigenvalue parameters are given by

$$
\left.\begin{array}{ccc}
p_{1}=-1.1, & p_{2}-3.5, & p_{3}=5.5 \\
q_{1}=-1.1, & q_{2}=3.5, & q_{3}=-11.0 .
\end{array}\right\}
$$

Now we add a small perturbation $\delta p_{1}=0.055$, and $\delta q_{1}=-0.055$ to obtain

$$
\left.\begin{array}{l}
p_{1}^{\prime}=-1.045, \quad p_{2}^{\prime}=3.5, \quad p_{3}^{\prime}=5.5 \\
q_{1}^{\prime}=-1.155, \quad q_{2}^{\prime}=3.5, \quad q_{3}^{\prime}=-11.0 .
\end{array}\right\}
$$

The corresponding dimensionless parameters are

$$
\left.\begin{array}{ccc}
\alpha=119.4383 & \beta=123.2917, & \gamma=-1, \\
a=-1.007900, & b=-1.008732, & \tau=-1.10751, \\
k=-1 . &
\end{array}\right\}
$$

By using the dimensionless parameters above, we obtain the attractor shown in Fig. 10.6(b) which is qualitatively similar to the attractor in Fig. 1(b) of (35). 


\subsection{Example from Dmitriev and Kislov}

In the oscillator system studied in (36-38) the nonlinearity is a cubic polynomial which becomes constant in an outer region. This can again be approximated by a piecewise-linear function, if we ignore the outer region.

The state equations are

$$
\left.\begin{array}{l}
\dot{x}=y \\
\dot{y}=-x-\delta y+z \\
\dot{z}=\gamma(F(x)-z)-\sigma y
\end{array}\right\}
$$

where

$$
F(x)= \begin{cases}0.528 \alpha & \text { if } \quad x<-1.2 \\ \alpha x\left(1-x^{2}\right) & \text { if } \quad-1.2<x<1.2 \\ -0.528 \alpha & \text { if } x>1.2\end{cases}
$$

The equilibrium points are given by

$$
\begin{array}{r}
y=0 \\
-x-\delta y+z=0 \\
\gamma(F(x)-z)-\sigma y=0 .
\end{array}
$$

Case 1. When $x<-1.2$, then

$$
\begin{array}{r}
y=0 \\
-x-\delta y+z=0 \\
\gamma(0.528 \alpha-z)-\sigma y=0,
\end{array}
$$

so we have

$$
\begin{aligned}
& x=0.528 \alpha \quad\left(\alpha=\frac{x}{0.528}<\frac{-1.2}{0.528}=-2.2727\right) \\
& y=0 \\
& z=0.528 \alpha .
\end{aligned}
$$

Case 2. When $-1.2<x<1.2$, then

$$
\begin{array}{r}
y=0 \\
-x-\delta y+z=0 \\
\gamma\left(\alpha x\left(1-x^{2}\right)-z\right)-\sigma y=0,
\end{array}
$$

so we have $x=0, x= \pm \sqrt{(\alpha-1) / \alpha}, \alpha \geqslant 1$. The three equilibrium points are $(\sqrt{(\alpha-1) / \alpha}, 0, \sqrt{(\alpha-1) / \alpha}),(0,0,0)$, and $(-\sqrt{(\alpha-1) / \alpha}, 0,-\sqrt{(\alpha-1) / \alpha})$. 
L. Pivka et al.

Case 3. When $x>1.2$, then

$$
\begin{array}{r}
y=0 \\
-x-\delta y+z=0 \\
\gamma(-0.528 x-z)-\sigma y=0,
\end{array}
$$

so we have

$$
\begin{aligned}
& x=-0.528 \alpha \quad\left(\alpha=\frac{x}{-0.528}<\frac{1.2}{-0.528}=-2.2727\right) \\
& y=0 \\
& z=-0.528 \alpha .
\end{aligned}
$$

We will consider values of $\alpha$ where there are no equilibrium points in the outer regions $(|x|>1.2)$. From Eq. (27), at the origin the Jacobian matrix is

$$
M_{0}=\left[\begin{array}{ccc}
0 & 1 & 0 \\
-1 & -\delta & 1 \\
x \gamma & -\sigma & -\gamma
\end{array}\right]
$$

At the two other equilibrium points, the Jacobian matrix is

$$
M_{ \pm}=\left[\begin{array}{ccc}
0 & 1 & 0 \\
-1 & -\delta & 1 \\
\alpha \gamma\left[1-3\left(\sqrt{\frac{\alpha-1}{\alpha}}\right)^{2}\right] & -\sigma & -\gamma
\end{array}\right] .
$$

Let $\alpha=16, \delta=0.43, \sigma=0.71$, and $\gamma=0.1$. We have

$$
M_{ \pm}=\left[\begin{array}{ccc}
0 & 1 & 0 \\
-1 & -0.43 & 1 \\
-2.9 & -0.71 & -0.1
\end{array}\right]
$$

with eigenvalues

$$
v_{1}=-1.18562, v_{2}=0.32781+j 1.55655, v_{3}=0.32781-j 1.55655
$$

and

$$
M_{0}=\left[\begin{array}{ccc}
0 & 1 & 0 \\
-1 & -0.43 & 1 \\
1.6 & -0.71 & -0.1
\end{array}\right]
$$

with eigenvalues

$$
\mu_{1}=0.61184, \mu_{2}=-0.57092+j 1.45797, \mu_{3}=-0.57092-j 1.45797 .
$$

So, the corresponding equivalent eigenvalue parameters are given by 


$$
\left.\begin{array}{ccc}
p_{1}=-0.530000, & p_{2}=1.7530, & p_{3}=1.5000 \\
q_{1}=-0.530000, & q_{2}=1.75299, & q_{3}=-3.0000 .
\end{array}\right\}
$$

Again we add a small perturbation, $\delta p_{1}=0.00265, \delta q_{1}=-0.00265$, to obtain

$$
\left.\begin{array}{l}
p_{1}^{\prime}=-0.52735, \quad p_{2}^{\prime}=1.7530, \quad p_{3}^{\prime}=1.5000 \\
q_{1}^{\prime}=-0.53265, \quad q_{2}^{\prime}=1.75299, \quad q_{3}^{\prime}=-3.0000 .
\end{array}\right\}
$$

These equivalent eigenvalues correspond to the following set of perturbed eigenvalues:

$$
\left.\begin{array}{c}
\mu_{1}^{\prime}=0.61212, \quad \mu_{2}^{\prime}=-0.56974+j 1.45805, \quad \mu_{3}^{\prime}=-0.56974-j 1.45805 \\
v_{1}^{\prime}=-1.18641, \quad v_{2}^{\prime}=0.32688+j 1.55621, \quad v_{3}^{\prime}=0.32688-j 1.55621 .
\end{array}\right\}
$$

The corresponding dimensionless parameters are

$$
\left.\begin{array}{ccc}
\alpha=2971.482, & \beta=2978.630, & \gamma=-0.99647, \\
a=-1.00033354, & b=-1.00033688, & \tau=-0.533989, \\
k=-1 . &
\end{array}\right\}
$$

By using the dimensionless parameters above, we obtain the attractor shown in Fig. 10.4(b).

For the convenience of the reader, we show in tabular format (see Table II) the parameters of several systems in $\mathscr{C}$ and the parameters of corresponding Chua's oscillators that generate qualitatively similar behavior. The matrix $\tilde{\mathbf{T}}$ defines the equivalence between the system in $\mathscr{C}$ and Chua's oscillator as follows:

$$
\mathbf{x}=\tilde{\mathbf{T}}\left(\begin{array}{c}
\tilde{x} \\
\tilde{y} \\
\tilde{z}
\end{array}\right)
$$

where $\tilde{x}, \tilde{y}, \tilde{z}$ are the state variables of the dimensionless Chua's oscillator. The regions $D_{+}, D_{-}$and $D_{0}$ in Chua's oscillator correspond to $\tilde{x} \geqslant 1, \tilde{x} \leqslant-1$, and $|\tilde{x}| \leqslant 1$, respectively. In Fig. 10 we show the attractors of the systems and the attractors of corresponding Chua's oscillators.

\section{Generation of Wave Phenomena in CNN Arrays of Chua's Oscillators}

The appearance of rotating spiral waves has been observed in many chemical and biological processes, including those in the cardiac muscle (39), retinae (40), and chemical oscillators such as the Belousov-Zhabotinsky reaction (41). Most of these systems have been successfully modeled by continuum models via partial differential equations. However, the above phenomena can be reproduced (42) more efficiently by using CNNs of discrete coupled cells. For the purpose of generating spiral waves we consider the nonlinear system of coupled Chua's oscillators (written in dimensionless form, with $k=1$ )

$$
\dot{x}_{i, j}=\alpha\left(y_{i, j}-x_{i, j}-f\left(x_{i, j}\right)\right)+D\left[x_{i+1, j}+x_{i-1, j}+x_{i, j+1}+x_{i, j-1}-4 x_{i, j}\right]
$$


L. Pivka et al.

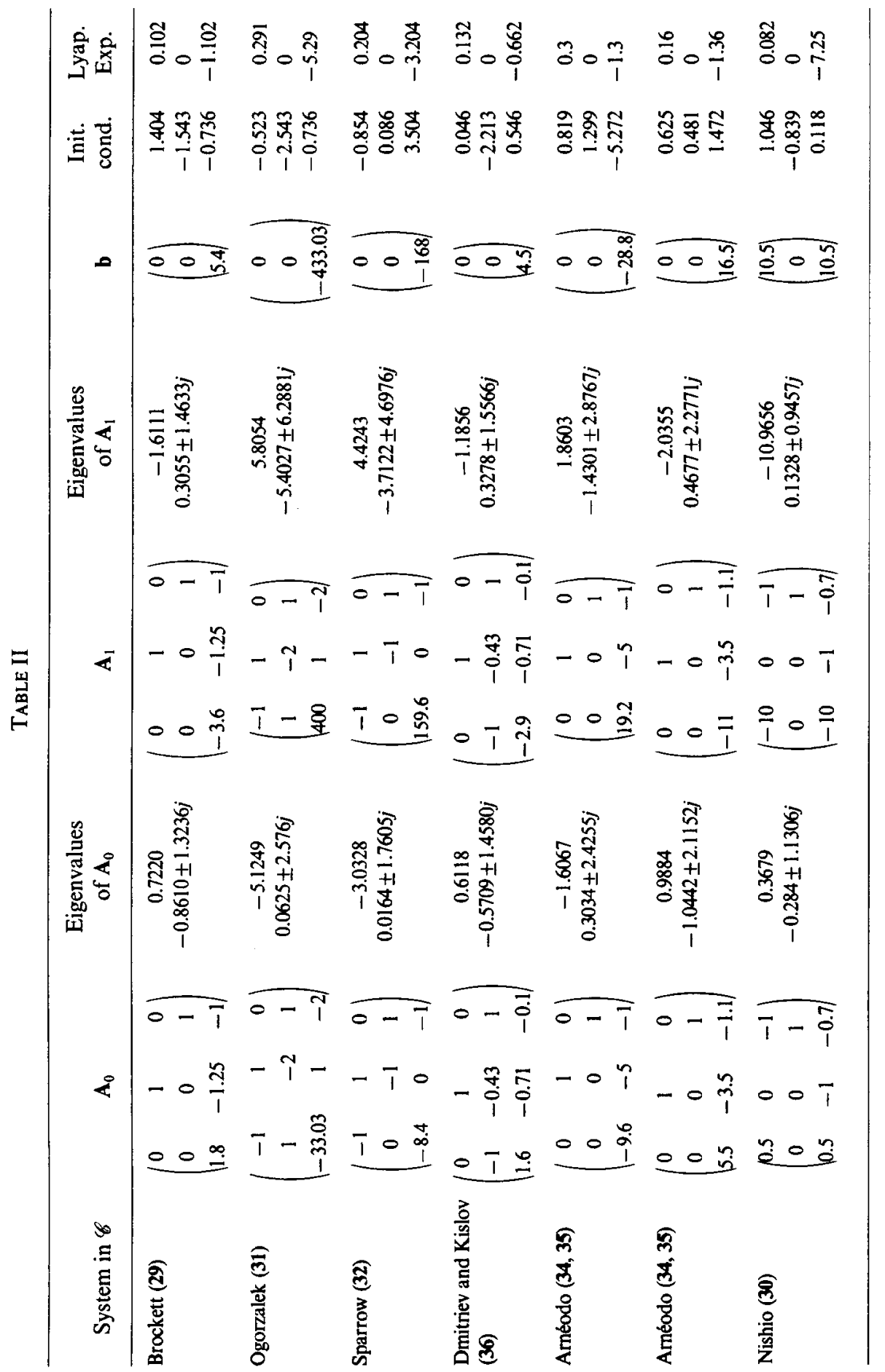




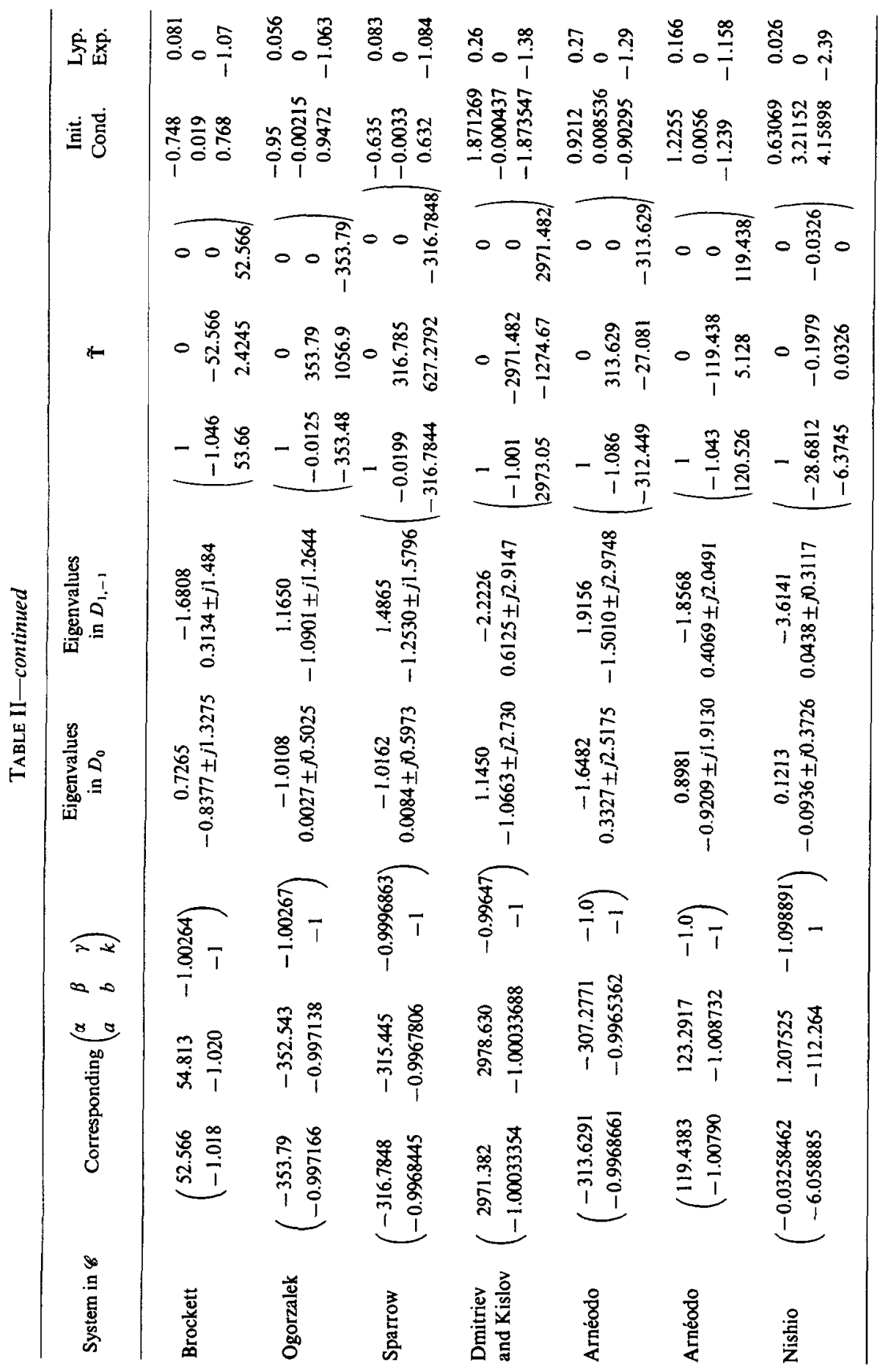




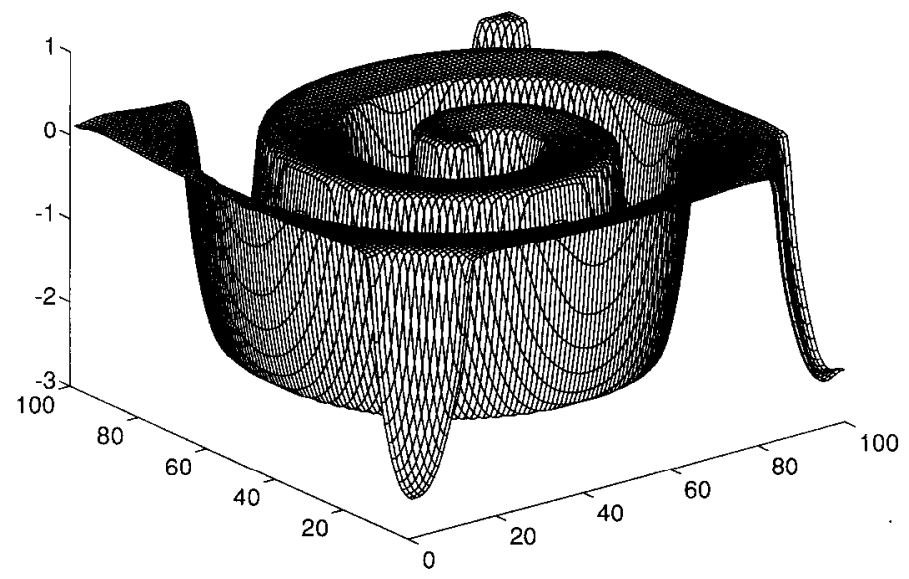

F1G. 11. Spiral wave generated in a $100 \times 100$ array of identical Chua's oscillators.

$$
\begin{aligned}
& \dot{y}_{i_{j}}=x_{i_{i}}-y_{i_{i}}+z_{i, j} \quad(i, j=1,2, \ldots, l) \\
& \dot{z}_{i_{i, j}}=-\beta y_{i, j}-\gamma z_{i, j} .
\end{aligned}
$$

The nonsymmetric three-segment PWL function $f(x)$ is given by

$$
f(x)=(1 / 2)\left[\left(s_{1}+s_{2}\right) x+\left(s_{0}-s_{1}\right)\left(\left|x-B_{1}\right|-\left|B_{1}\right|\right)+\left(s_{2}-s_{0}\right)\left(\left|x-B_{2}\right|-\left|B_{2}\right|\right)\right]
$$

where in this case we consider breakpoints $B_{1}=-1, B_{2}=\left(s_{0}-s_{1}\right) /\left(s_{0}-s_{2}\right)$. The fundamental regime in each individual cell is a cyclical one and is achieved by choosing the parameter values, e.g. as follows: $\alpha=10, \beta=0.334091, \gamma=0$, $s_{1}=0.020706, s_{0}=-0.921, s_{2}=15$. The strong asymmetry of function $f$ provides for a high-relaxation character of the limit cycle, which is necessary for the dynamics to be stable. Figure 11 shows a fully evolved spiral with the diffusion coefficient $D=5$. Many other interesting phenomena and patterns can be generated in 2- and 3-dimensional arrays, for example autowaves (43), Turing patterns (44), and scroll waves (45).

\section{Conclusions}

Chua's circuit has proven to be an excellent paradigm for the generation of a multitude of different dynamical phenomena, and can thus obviate the need to consider many different models to simulate those phenomena. Chua's oscillator has unified the nonlinear dynamics of the entire 12-parameter family of piecewiselinear vector fields into a single system defined by Eq. (2), hence it is not necessary for beginners in nonlinear dynamics to study all those papers with diverse notations and jargons.

Even more significantly, arrays of Chua's circuits appear to be a suitable candidate for important applications ranging from image processing to the simulations 
of biological processes. The building of the monolithic IC chip of Chua's circuit is an important step towards building large arrays via VLSI technology, and will make it possible to reproduce, in real time, almost all reaction-diffusion situations described in the literature with a relatively simple low-cost system.

\section{Acknowledgements}

This work was supported in part by the Office of Naval Research under grant N0001489-J-1402, by the National Science Foundation under grant MIP 86-14000, and by the Joint Services Electronics Program, Contract Number F49620-94-C-0038. The United States Government is authorized to reproduce and distribute reprints for governmental purposes notwithstanding any copyright notation hereon.

\section{References}

(1) E. N. Lorenz, "Deterministic non-periodic flows", J. Atmos. Sci., Vol. 20, pp. 130 $141,1963$.

(2) M. L. Cartwright and J. E. Littlewood, "On nonlinear differential equations of the second order", J. London Math. Soc., Vol. 20, pp. 180-189, 1945.

(3) K. Wegmann and O. E. Roessler, "Different kinds of chaotic oscillations in the Belousov-Zhabotinskii reaction", Z. Naturforsch., Vol. 33a, pp. 1179-1183, 1978.

(4) A. N. Sharkovskii, "Coexistence of cycles of a continuous map of a line into itself", Ukrainian Math. J., Vol. 16, pp. 61-71, 1964.

(5) L. P. Shilnikov, "A case of the existence of a denumerable set of periodic motions", Soviet Math. Dokl., Vol. 6, pp. 163-166, 1965.

(6) L. P. Shilnikov, "Existence of a countable set of periodic motions in a four-dimensional space in an extended neighborhood of a saddle-focus", Soviet Math. Dokl., Vol. 8 , pp. 54-58, 1967.

(7) S. Smale, "Differentiable dynamical systems", Bull. Am. Math. Soc., Vol. 73, pp. 747$817,1967$.

(8) L. O. Chua, "Global unfolding of Chua's circuit", IEICE Trans. Fund. Electr. Comm. Comput. Sci., Vol. E76-A, pp. 704-734, 1993.

(9) L. O. Chua, "A simple ODE with more than 20 strange attractors", Memorandum No. UCB/ERL M92/141, UC Berkeley, 1992.

(10) L. Pivka and V. Špány, "Boundary surfaces and basin bifurcations in Chua's circuit", J. Circuits Syst. Comput., Vol. 3, pp. 441-470, 1993.

(11) L. O. Chua, C. W. Wu, A. S. Huang and G.-Q. Zhong, "A universal circuit for studying and generating Chaos, Parts I, II", IEEE Trans. Circuits \& Syst.-I: Fund. Theory Appl., Vol. 40, pp. 732-761, (1993).

(12) R. Lozi and S. Ushiki, "Co-existing attractors in Chua's circuit: Accurate analysis of bifurcation and attractors", Int. J. Bifurcation Chaos, Vol. 1, pp. 923-926, 1991.

(13) L. Duchesne, "Using characteristic multiplier loci to predict bifurcation phenomena and chaos-a tutorial", IEEE Trans. Circuits \& Syst.-I: Fund. Theory Appl., Vol. 40, pp. 683-688, 1993.

(14) V. S. Anishchenko, A. B. Neiman and L. O. Chua, "Chaos-chaos intermittency and l/f noise in Chua's circuit", Int. J. Bifurcation Chaos, Vol. 4, pp. 99-107, 1994.

(15) V. S. Anishchenko, M. A. Safonova and L. O. Chua, "Stochastic resonance in the nonautonomous Chua's circuit", J. Circuits Syst. Comput., Vol. 3, pp. 553-578, 1993.

(16) K. S. Halle, L. O. Chua, V. S. Anishchenko and M. A. Safonova, "Signal amplification 
via chaos: Experimental evidence", Int. J. Bifurcation Chass, Vol. 2, pp. 1011-1020, 1992.

(17) A. I. Khibnik, D. Roose and L. O. Chua, "On periodic orbits and homoclinic bifurcations in Chua's circuit with smooth nonlinearity", Int. J. Bifurcation Chaos, Vol. 3, pp. 363-384, 1993.

(18) G.-Q. Zhong, "Implementation of Chua's circuit with a cubic nonlinearity", Memorandum No. UCB/ERL M94/42, UC Berkeley, USA, 1994.

(19) L. O. Chua and I. Tichonicky, "1D map for the double scroll family", IEEE Trans. Circuits \& Syst., Vol. 38, pp. 233-243, 1991.

(20) M. J. Feigenbaum, "The universal metric properties of nonlinear transformations", $J$. Stat. Phys., Vol. 21, pp. 669-706, 1979.

(21) R. Madan and C. W. Wu, "Introduction to experimental chaos using Chua's circuit", in "Chua's Circuit: A Paradigm for Chaos", World Scientific, Singapore, pp. 5989, 1993.

(22) A. P. Kuznetsov, S. P. Kuznetsov, I. R. Sataev and L. O. Chua, "Two-parameter study of transition to chaos in Chua's circuit: Renormalization group, universality and scaling", Int. J. Bifurcation Chaos, Vol. 3, pp. 943-962, 1993.

(23) I. Kan and J. A. Yorke, "Antimonotonicity-concurrent creation and annihilation of periodic orbits", Bull. Am. Math. Soc., Vol. 23, pp. 469-476, 1990.

(24) Lj. Kočarev, K. S. Halle, K. Eckert and L. O. Chua, "Experimental observation of antimonotonicity in Chua's circuit", Int. J. Bifurcation Chas, Vol. 3, pp. 1051-1055, 1993.

(25) L. Pivka, A. L. Zheleznyak and L. O. Chua, "Arnol'd tongues, the devil's staircase, and self-similarity in the driven Chua's circuit", Int. J. Bifurcation Chaos, Vol. 4, pp. 1743-1753, 1994.

(26) V. C. Anishchenko, T. E. Vadivasova, D. E. Postnov, O. V. Sosnovtseva, L. O. Chua and C. W. Wu, "Dynamics of the non-autonomous Chua's circuit", to appear in Int. J. Bifurcation Chaos, Vol. 5, 1995.

(27) M. Itoh and H. Murakami, "Experimental study of forced Chua's oscillator", Int. $J$. Bifurcation Chaos, Vol. 4, pp. 1721-1742, 1994.

(28) P. Deregel, "Chua's oscillator: A zoo of attractors", J. Circuits Syst. Comput., Vol. 3, pp. 309-359, 1993.

(29) R. W. Brockett, "On conditions leading to chaos in feedback systems", in "Proc. IEEE Conf. Decision Contr.”, pp. 932-936, IEEE, Piscataway, NJ, 1982.

(30) Y. Nishio, N. Inaba and S. Mori, "Chaotic phenomena in an autonomous circuit with nonlinear inductor", in "Proc. ISCAS", IEEE, Piscataway, NJ, 1990.

(31) M. J. Ogorzalek, "Order and chaos in a third-order RC ladder network with nonlinear feedback", IEEE Trans. Circuits \& Syst., Vol. 36, pp. 1221-1230, 1989.

(32) C. T. Sparrow, "Chaos in three-dimensional single loop feedback system with a piecewise-linear feedback function", J. Math. Anal. Appl., Vol. 83, pp. 275-291, 1981.

(33) P. Coullet, C. Tresser and A. Arnéodo, "Transition to stochasticity for a class of forced oscillators", Phys. Lett., Vol. 72A, pp. 268-270, 1979.

(34) A. Arnéodo, P. Coullet and C. Tresser, "Possible new strange attractors with spiral structure", Comm. Math. Phys., Vol. 79, pp. 573-579, 1981.

(35) A. Arnéodo, P. Coullet and E. A. Spiegel, "Chaos in a finite macroscopic system", Phys. Lett., Vol. 92A, pp. 369-373, 1982.

(36) A. S. Dmitriev and V. Y. Kislov, "Stochastic oscillations in a self-excited oscillator with a first-order inertial delay," Radiotekhnika i elektronika, Vol. 29, pp. 2389, 1984 (in Russian).

(37) A. Volkovskii and N. F. Rul'kov, "Use of one-dimensional mapping for an exper- 
imental study of the stochastic dynamics of an oscillator", Sov. Tech. Phys. Lett., Vol. 14, pp. 656-658, 1988.

(38) N. F. Rul'kov, A. R. Volkovskii, A. Rodriguez-Lozano, E. D. Rio and M. G. Velarde, "Mutual synchronization of chaotic self-oscillators with dissipative coupling", Int. J. Bifurcation Chaos, Vol. 2, pp. 669-676, 1992.

(39) M. A. Allesie, F. I. M. Bonke and T. Y. G. Schopman, "Circus movement in rabbit atrial muscle as a mechanism in tachycardia", Circulation Res., Vol. 33, pp. 54-62, 1973.

(40) J. Bures, V. I. Koroleva and N. A. Gorelova, "Leao's spreading depression, an example of diffusion-mediated propagation of excitation in the central nervous system", in "Autowaves and Structures Far from Equilibrium" (edited by V. I. Krinsky), pp. 180-183, Springer-Verlag, 1984.

(41) S. C. Müller, T. Plesser and B. Hess, "Two-dimensional spectrophotometry of spiral wave propagation in the Belousov-Zhabotinskii reaction, I. Experiments and digital representation; II. Geometric and kinematic parameters", Physica, Vol. D24, pp. 71-96, 1987.

(42) A. Pérez-Muñuzuri, V. Pérez-Muñuzuri, V. Pérez-Villar and L. O. Chua, "Spiral waves on a two-dimensional array of nonlinear circuits", IEEE Trans. Circuits \& Syst., I: Fund Theory Appl., Vol. 40, pp. 872-877, 1993.

(43) V. Pérez-Muñuzuri, V. Pérez-Villar and L. O. Chua, "Autowaves for image processing on a two-dimensional CNN array of excitable nonlinear circuits: Flat and wrinkled labyrinths", IEEE Trans. Circuits \& Syst., Vol. 40, pp. 174-181, 1993.

(44) V. Pérez-Muñuzuri, M. Gómez-Gesteira, A. Pérez-Muñuzuri, L. O. Chua and V. Pérez-Villar, "Sidewall forcing of hexagonal Turing patterns: Rhombic patterns", to appear in Physica D.

(45) L. Pivka, A. L. Zheleznyak, C. W. Wu and L. O. Chua, "On the generation of scroll waves in a three-dimensional discrete active medium", Int. J. Bifurcation Chaos, Vol. 5, pp. 313-320, 1995. 